\title{
Scalar Cosmological Perturbations
}

\author{
Claes Uggla* \\ Department of Physics, \\ University of Karlstad, S-651 88 Karlstad, Sweden \\ JOHN WAINWRIGHT ${ }^{\dagger}$ \\ Department of Applied Mathematics, \\ University of Waterloo, Waterloo, ON, N2L 3G1, Canada
}

March 7, 2018

\begin{abstract}
Scalar perturbations of Friedmann-Lemaitre cosmologies can be analyzed in a variety of ways using Einstein's field equations, the Ricci and Bianchi identities, or the conservation equations for the stress-energy tensor, and possibly introducing a timelike reference congruence. The common ground is the use of gauge invariants derived from the metric tensor, the stress-energy tensor, or from vectors associated with a reference congruence, as basic variables. Although there is a complication in that there is no unique choice of gauge invariants, we will show that this can be used to advantage.

With this in mind our first goal is to present an efficient way of constructing dimensionless gauge invariants associated with the tensors that are involved, and of determining their inter-relationships. Our second goal is to give a unified treatment of the various ways of writing the governing equations in dimensionless form using gauge-invariant variables, showing how simplicity can be achieved by a suitable choice of variables and normalization factors. Our third goal is to elucidate the connection between the metric-based approach and the so-called $1+3$ gauge-invariant approach to cosmological perturbations. We restrict our considerations to linear perturbations, but our intent is to set the stage for the extension to second order perturbations.
\end{abstract}

PACS numbers: 04.20.-q, 98.80.-k, 98.80.Bp, 98.80.Jk

*Electronic address: claes.uggla@kau.se

$\dagger$ Electronic address: jwainwri@uwaterloo.ca 


\section{Introduction}

Currently, increasingly accurate observations are driving theoretical cosmology towards more sophisticated models of matter and the study of possible nonlinear deviations from FL cosmology 1 Motivated by this state of affairs, in a recent paper (Uggla and Wainwright (2011), hereafter referred to as UW), we initiated a program of research whose long term goal is to provide a general but concise description of nonlinear perturbations of FL cosmologies that will reveal the structure of the governing equations, and hence facilitate their analysis. In furthering this goal one is faced with making three choices. First, there is the choice of gauge-invariant variables: the work of Bardeen (1980) made clear that there is no unique choice. Second, the use of dimensionless variables invariably leads to physical insight via the choice of a suitable normalizing factor or factors. Third, there is the choice of how to formulate the governing equations: Einstein's field equations, the Ricci and Bianchi identities, the conservation equation for stress-energy, or other matter equations. In this paper we systematically consider these three choices, working for the moment within the framework of linear perturbation theory.

Our first goal in this paper is to present an efficient way of constructing dimensionless gauge invariants associated with the metric tensor, the stress-energy tensor, or other structures that may be introduced, and of determining their interrelationships. We use the method of Nakamura (2003), adapted as in UW to create dimensionless gauge invariants.

Our second goal is to give a unified treatment of the various ways of writing the governing equations in dimensionless form using gauge-invariant variables within the framework of the metric-based approach to cosmological perturbations 2 In UW we gave the linearized Einstein equations in two forms, which we referred to as the Poisson form, associated with the work of Bardeen (1980), and the uniform curvature form, associated with the work of Kodama and Sasaki (1984). In the present paper we derive the linearized conservation equations for the stress-energy tensor and by expressing them in terms of suitable gauge invariants, give an alternative description of the dynamics of scalar perturbations as a system of two first order (in time) partial differential equations. We also include the case where the source has multiple components. In addition, by using the inter-relationships between the different gauge invariants, we are able to give a unified description of the various "conserved quantities" that are associated with long wavelength scalar perturbations.

Our third goal is to elucidate the connection between the metric-based approach and the so-called $1+3$ gauge-invariant approach to cosmological perturbations, which was developed with the goal of circumventing the gauge difficulties associ-

\footnotetext{
${ }^{1}$ We follow the nomenclature of Wainwright and Ellis (1997): a Friedmann-Lemaitre (FL) cosmology is a Robertson-Walker (RW) geometry that satisfies Einstein's field equations.

${ }^{2}$ By this we mean the standard approach to cosmological perturbations in which one formulates the governing equations in terms of gauge-invariant variables associated with the perturbed metric tensor and the perturbed stress-energy tensor, using local coordinates.

${ }^{3}$ The $1+3$ gauge-invariant approach uses variables that are apriori gauge-invariant at first order due to the Stewart-Walker lemma and are defined using the $1+3$ covariant description of GR, which is based on a preferred timelike congruence. This approach is growing in popularity. For a recent treatment in depth we refer to Tsagas, Challinor and Maartens (2008).
} 
ated with scalar perturbations (Hawking (1966), Ellis and Bruni (1989) and Ellis, Bruni and Hwang (1990)). The $1+3$ approach is formulated independently of the metric-based approach 4 and indeed there is a significant gap between the two approaches. In the metric-based approach it is customary to expand the metric and other basic variables in terms of a power series in a perturbation parameter as in UW, since this clarifies the linearization procedure and permits one to extend the analysis to higher order perturbations. In this respect the metric-based approach is analogous to standard elementary perturbation procedures in physics and engineering. On the other hand, the $1+3$ approach is not formulated as a conventional perturbation procedure, and relies instead on deriving exact evolution equations which are then linearized by dropping products of first order terms. In this paper we will reformulate the $1+3$ approach so as to bridge the above-mentioned gap.

The plan of the paper is as follows. In section 2 we give the metric and stressenergy gauge invariants and specify the four gauge choices and the two normalizations that we will use. In section 3 we discuss the equations for scalar perturbations that arise from the linearization of the conservation law for the stress-energy tensor, and their relation with the linearized Einstein equations. The details of the derivation, which makes use of the Replacement Principle in Appendix A, are given in Appendix B. In section 4 we give a concise derivation of the so-called conserved quantities in gauge-invariant form. In section 5 we introduce the basic variables in the $1+3$ gauge-invariant approach to scalar perturbations and derive the governing equations, which we then relate to the corresponding equations in the metric-based approach. The details are relegated to Appendix [C. Section 6 contains a brief summary and discussion.

\section{Gauge invariants and gauge fields}

We begin by describing a dimensionless version of Nakamura's method for constructing gauge invariants (see Nakamura (2007), equations (2.19), (2.23) and (2.26), and UW, section 2.1, for a brief introduction). Consider a family of tensor fields $A(\epsilon)$ and a background scalar $\lambda$ having dimension length such that $\lambda^{n} A(\epsilon)$ is dimensionles: 5 . The change induced in the first order perturbation ${ }^{(1)} A$ by a gauge transformation generated by a dimensionless vector field $\xi$ on the background can be expressed using the Lie derivative $£$ :

$$
\Delta^{(1)} A=£_{\xi}^{(0)} A,
$$

(see, for example, Bruni et al (1997), equation (1.2)). Let $X$ be a dimensionless vector field that satisfies

$$
\Delta X^{a}=\xi^{a} .
$$

It follows that the dimensionless object defined by

$$
\mathbf{A}[X]:=\lambda^{n}\left({ }^{(1)} A-£_{X}{ }^{(0)} A\right),
$$

\footnotetext{
${ }^{4}$ In the $1+3$ approach the metric tensor is not used as a dynamical variable and local coordinates are not introduced, in contrast to the metric-based approach.

${ }^{5}$ See UW, footnote 9 , for a discussion and references about allocation of dimensions.
} 
is gauge-invariant. We say that $\mathbf{A}[X]$ is the gauge invariant associated with ${ }^{(1)} A$ by $X$-compensation. Since a choice of $X$ yields a set of gauge-invariant variables that are associated with a specific fully fixed gauge we refer to $X$ as the gauge field. In this paper we will use two choices for the normalization factor $\lambda$ : if $A$ is a geometric quantity, we will use $\lambda=a$, where $a$ is the background scale factor, while if $A$ is a matter quantity we will use $\lambda=\mathcal{M}$, where $\mathcal{M}$ is defined 6 by (15). In the latter case we will denote $\mathbf{A}[X]$ by $\mathbb{A}[X]$.

\subsection{Metric gauge invariants}

Given a 1-parameter family of metrics $g_{a b}(\epsilon)$, where $\epsilon$ is a perturbation parameter and $g_{a b}(0)$ is a Robertson-Walker (RW) metric, we define a dimensionless conformal metric $\bar{g}_{a b}(\epsilon)$ according to

$$
g_{a b}(\epsilon)=a^{2} \bar{g}_{a b}(\epsilon)
$$

where $a$ is the scale factor of the RW metric. We expand $\bar{g}_{a b}(\epsilon)$ in powers of $\epsilon$ :

$$
\bar{g}_{a b}(\epsilon)={ }^{(0)} \bar{g}_{a b}+\epsilon^{(1)} \bar{g}_{a b}+\ldots,
$$

and label the unperturbed metric and (linear) metric perturbation according to

$$
\gamma_{a b}:={ }^{(0)} \bar{g}_{a b}=\bar{g}_{a b}(0), \quad f_{a b}:={ }^{(1)} \bar{g}_{a b}=\left.\frac{\partial \bar{g}_{a b}}{\partial \epsilon}\right|_{\epsilon=0} .
$$

In order to construct a gauge field $X$ that satisfies (2), using only the metric, we need to decompose the metric perturbation $f_{a b}$ into scalar, vector and tensor modes. Relative to a local coordinate system 7 we introduce the notation

$$
\begin{aligned}
& f_{00}=-2 \varphi, \\
& f_{0 i}=\mathbf{D}_{i} B+B_{i}, \\
& f_{i j}=-2 \psi \gamma_{i j}+2 \mathbf{D}_{i} \mathbf{D}_{j} C+2 \mathbf{D}_{(i} C_{j)}+2 C_{i j},
\end{aligned}
$$

where the vectors $B_{i}$ and $C_{i}$ and the tensor $C_{i j}$ satisfy

$$
\mathbf{D}^{i} B_{i}=0, \quad \mathbf{D}^{i} C_{i}=0, \quad C_{i}^{i}=0, \quad \mathbf{D}^{i} C_{i j}=0,
$$

where $\mathbf{D}_{i}$ is the spatial covariant derivative associated with $\gamma_{i j}$. We can satisfy the spatial part $\Delta X^{i}=\xi^{i}$ of the requirement (2) by choosing

$$
X_{i}=\mathbf{D}_{i} C+C_{i}
$$

(UW, section 2.2), which we will take to be our default choice for $X_{i}$. With this choice, the components of the gauge invariant $\mathbf{f}_{a b}[X]$ associated with the metric perturbation $f_{a b}$ by $X$-compensation, are given by (UW, equations (21), (23) and (25))

$$
\begin{aligned}
\mathbf{f}_{00}[X] & =-2 \Phi[X], \\
\mathbf{f}_{0 i}[X] & =\mathbf{D}_{i} \mathbf{B}[X]+\mathbf{B}_{i}, \\
\mathbf{f}_{i j}[X] & =-2 \Psi[X] \gamma_{i j}+2 \mathbf{C}_{i j} .
\end{aligned}
$$

\footnotetext{
${ }^{6}$ This choice is motivated in section 2.2 .
}

${ }^{7}$ See UW equations (10) and (11). 
where

$$
\begin{gathered}
\Phi[X]:=\varphi-\left(\partial_{\eta}+\mathcal{H}\right) X^{0}, \quad \Psi[X]:=\psi+\mathcal{H} X^{0}, \quad \mathbf{B}[X]:=B-\partial_{\eta} C+X^{0}, \\
\mathbf{B}_{i}:=B_{i}-\partial_{\eta} C_{i}, \quad \mathbf{C}_{i j}:=C_{i j} .
\end{gathered}
$$

In equation (9a), $\mathcal{H}$ is the dimensionless Hubble scalar, defined by 8

$$
\mathcal{H}:=\frac{a^{\prime}}{a} .
$$

The quantities $\mathbf{B}_{i}$ and $\mathbf{C}_{i j}$, which describe the vector mode and tensor mode of the perturbation respectively, are intrinsic metric gauge invariant 9 that are independent of the gauge field $X$. In contrast the gauge invariants $\Phi[X], \Psi[X]$ and $\mathbf{B}[X]$, which describe the scalar mode, depend on the choice of $X^{0}$ but not on the choice of the spatial gauge field $X^{i}$. Of course, if we leave $X^{i}$ arbitrary then $\mathbf{f}_{a b}[X]$ contains additional terms and its components are given by

$$
\begin{aligned}
\mathbf{f}_{00}[X] & =-2 \Phi[X] \\
\mathbf{f}_{0 i}[X] & =\mathbf{D}_{i} \mathbf{B}[X]+\mathbf{B}_{i}+\partial_{\eta} \mathbf{Z}_{i}[X] \\
\mathbf{f}_{i j}[X] & =-2 \Psi[X] \gamma_{i j}+2 \mathbf{C}_{i j}+2 \mathbf{D}_{(i} \mathbf{Z}_{j)}[X]
\end{aligned}
$$

where

$$
\mathbf{Z}_{i}[X]:=\mathbf{D}_{i} C+C_{i}-X_{i}
$$

Our default choice (7) for $X^{i}$ corresponds to $\mathbf{Z}_{i}[X]=0$.

\subsection{Stress-energy gauge invariants}

Consider a stress-energy tensor $T_{b}^{a}(\epsilon)$ that obeys the background symmetries, i.e., it is spatially homogeneous and isotropic:

$$
\mathbf{D}_{i}{ }^{(0)} T_{\beta}^{\alpha}=0, \quad{ }^{(0)} T_{i}^{0}={ }^{(0)} T_{0}^{i}=0, \quad{ }^{(0)} T_{j}^{i}=\frac{1}{3} \delta_{j}^{i}{ }^{(0)} T_{k}^{k} .
$$

We assume that $T_{b}^{a}(\epsilon)$ satisfies the conservation law ${ }^{\epsilon} \nabla_{b} T_{a}^{b}(\epsilon)=0$, which at zeroth order yields

$$
{ }^{(0)} \rho^{\prime}=-3 \mathcal{H}\left({ }^{(0)} \rho+{ }^{(0)} p\right)
$$

where

$$
{ }^{(0)} \rho=-{ }^{(0)} T_{0}^{0}, \quad{ }^{(0)} p=\frac{1}{3}{ }^{(0)} T_{k}^{k} .
$$

When constructing dimensionless gauge invariants it is necessary to choose a normalization factor. In Newtonian theory the dimensionless quantity $\delta \rho / \rho \equiv{ }^{(1)} \rho /{ }^{(0)} \rho$, where $\rho$ is the mass density, is used to describe structure formation. By analogy the same quantity is usually used in GR, but with $\rho$ being the mass-energy density instead. We propose that in GR a more natural normalization factor is the inertial

\footnotetext{
${ }^{8}$ Here and elsewhere we denote the derivative of a function $f(\eta)$ that depends only on $\eta$ by $f^{\prime}(\eta)$.

${ }^{9}$ Intrinsic gauge invariants were defined in UW, section 2.1, as gauge invariants constructed solely from a single tensor, in contrast to hybrid gauge invariants that are constructed from several tensors.
} 
mass-energy density ${ }^{(0)} \rho+{ }^{(0)} p$, since this is the quantity that appears instead of ${ }^{(0)} \rho$ in the relativistic energy-momentum conservation equations. As we will show in this paper normalizing with ${ }^{(0)} \rho+{ }^{(0)} p$ leads to a simpler description of scalar density perturbations when using matter variables. We shall refer to this type of normalization as inertial mass-density normalization or more briefly, as $\mathcal{M}$-normalization.

In order to implement the above idea we assume that the inertial mass-density ${ }^{(0)} \rho+{ }^{(0)} p$ in (14) is positive, and introduce a normalization factor with dimension length, defined by

$$
\mathcal{M}:=\left({ }^{(0)} \rho+{ }^{(0)} p\right)^{-1 / 2} .
$$

As in UW we introduce the notation

$$
\mathcal{A}_{T}:=a^{2}\left({ }^{(0)} \rho+{ }^{(0)} p\right), \quad \mathcal{C}_{T}^{2}:=\frac{{ }^{(0)} p^{\prime}}{{ }^{(0)} \rho^{\prime}}
$$

and in analogy with $\mathrm{UW}^{10}$ we define the following intrinsic gauge invariants associated with the stress-energy tensor 11 , using $\mathcal{M}$-normalization:

$$
\begin{aligned}
\hat{\mathbb{T}}_{j}^{i} & :=\mathcal{M}^{2}{ }^{(1)} \hat{T}^{i}{ }_{j} \\
\mathbb{T}_{i} & :=-\mathcal{M}^{2}\left(\mathbf{D}_{i}{ }^{(1)} T_{0}^{0}+3 \mathcal{H}^{(1)} T_{i}^{0}\right), \\
\mathbb{T} & :=\mathcal{M}^{2}\left(\mathcal{C}_{T}^{2(1)} T_{0}^{0}+\frac{1}{3}{ }^{(1)} T_{k}^{k}\right),
\end{aligned}
$$

where

$$
{ }^{(1)} \hat{T}_{j}^{i}:={ }^{(1)} T_{j}^{i}-\frac{1}{3} \delta^{i}{ }^{(1)} T_{k}^{k} .
$$

We also introduce the following gauge invariants by $X$-compensation 12 using $\mathcal{M}$ normalization:

$$
\begin{aligned}
& \mathbb{T}_{0}^{0}[X]:=\mathcal{M}^{2}\left({ }^{(1)} T_{0}^{0}\right)-3 \mathcal{H} X^{0} \\
& \mathbb{T}^{0}{ }_{i}[X]:=\mathcal{M}^{2}{ }^{(1)} T_{i}^{0}+\mathbf{D}_{i} X^{0} .
\end{aligned}
$$

It follows from (17b) and (19) that

$$
\mathbb{T}_{i}=-\left(\mathbf{D}_{i} \mathbb{T}_{0}^{0}[X]+3 \mathcal{H} \mathbb{T}_{i}^{0}[X]\right)
$$

Note that stress-energy gauge invariants depend only on the choice of $X^{0}$, not on $X^{i}$.

In analogy with UW (see equations (50)) we decompose the matter gauge invariants $\hat{\mathbb{T}}_{j}^{i}, \mathbb{T}_{i}, \mathbb{T}, \mathbb{T}_{i}^{0}[X]$ and $\mathbb{T}_{0}^{0}$ into scalar, vector, and tensor modes and label them as follows:

$$
\begin{aligned}
\hat{\mathbb{T}}_{j}^{i} & =\mathbf{D}_{j}^{i} \bar{\Pi}+2 \gamma^{i k} \mathbf{D}_{(k} \bar{\Pi}_{j)}+\bar{\Pi}_{j}^{i}, \\
\mathbb{T}_{i} & =\mathbf{D}_{i} \mathbb{D}+\mathbb{D}_{i}, \\
\mathbb{T} & =\bar{\Gamma} \\
\mathbb{T}_{i}^{0}[X] & =\mathbf{D}_{i} \mathbb{V}[X]+\mathbb{V}_{i}, \\
\mathbb{T}_{0}^{0}[X] & =-\mathbb{D}[X],
\end{aligned}
$$

\footnotetext{
${ }^{10}$ Replace $A$ by $T$ in equations (39) and (40) in UW.

${ }^{11}$ The $a$-normalized gauge invariants $\mathbf{T}_{b}^{a}$ in UW are related to the corresponding $\mathcal{M}$-normalized gauge invariants $\mathbb{T}^{a}{ }_{b}$ via $\mathbf{T}^{a}{ }_{b}=\mathcal{A}_{T} \mathbb{T}^{a}{ }_{b}$, where $\mathcal{A}_{T}=a^{2}\left({ }^{(0)} \rho+{ }^{(0)} p\right)=(a / \mathcal{M})^{2}$.

${ }^{12}$ Replace $A$ by $T$ in equations (38a) and (38b) in UW and multiply by $1 / \mathcal{A}_{T}=(\mathcal{M} / a)^{2}$. These equations also arise from (3) with $A$ replaced by $T$ and $\lambda=\mathcal{M}$.
} 
where

$$
\mathbf{D}^{i} \bar{\Pi}_{i}=0, \quad \bar{\Pi}_{k}^{k}=0, \quad \mathbf{D}_{i} \bar{\Pi}_{j}^{i}=0, \quad \mathbf{D}^{i} \mathbb{D}_{i}=0, \quad \mathbf{D}^{i} \mathbb{V}_{i}=0
$$

and

$$
\mathbf{D}_{i j}:=\mathbf{D}_{(i} \mathbf{D}_{j)}-\frac{1}{3} \gamma_{i j} \mathbf{D}^{2}, \quad \mathbf{D}^{2}:=\mathbf{D}^{i} \mathbf{D}_{i}
$$

It follows from (20) and (21) that

$$
\mathbb{D}=\mathbb{D}[X]-3 \mathcal{H} \mathbb{V}[X], \quad \mathbb{D}_{i}=-3 \mathcal{H} \mathbb{V}_{i}
$$

\subsection{Standard choices of the gauge field}

In order to eliminate the gauge freedom in the scalar mode, thereby determining the perturbed metric uniquely, we have to fully specify the gauge field $X$. We fix the spatial part $X^{i}$ of the gauge field $a b$ initio as in equation (7), leaving the temporal part $X^{0}$ to be specified. We observe that $X^{0}$ appears linearly and algebraically in the definitions of the gauge invariants:

$$
\Psi[X], \quad \mathbf{B}[X], \quad \mathbb{D}[X], \quad \mathbb{V}[X] .
$$

Note that $\Psi[X]$ and $\mathbf{B}[X]$ are defined by (9a), while $\mathbb{D}[X]$ and $\mathbb{V}[X]$ are given by 13

$$
\mathbb{D}[X]=-\mathcal{M}^{2(1)} T_{0}^{0}+3 \mathcal{H} X^{0}, \quad \mathbb{V}[X]=\mathcal{M}^{2} \mathbf{D}^{-2} \mathbf{D}^{i(1)} T_{i}^{0}+X^{0},
$$

as follows from (19) $,(21 \mathrm{~d}),(21 \mathrm{e})$ and (21f). We can thus determine $X^{0}$ uniquely by requiring that one of these four variables be zero. These choices in fact correspond to four of the commonly used gauges in cosmological perturbation theory 14

(i) Poisson gauge:

$$
\mathbf{B}\left[X_{\mathrm{p}}\right]=0 .
$$

(ii) Uniform curvature gauge:

$$
\Psi\left[X_{\mathrm{c}}\right]=0 .
$$

(iii) Total matter gauge:

$$
\mathbb{V}\left[X_{\mathrm{v}}\right]=0
$$

(iv) Uniform density gauge:

$$
\mathbb{D}\left[X_{\rho}\right]=0 .
$$

\footnotetext{
${ }^{13}$ In deriving the expression for $\mathbb{V}[X]$, we assume, as in $\mathrm{UW}$, that the inverse operator of $\mathbf{D}^{2}$ exists. In terms of the $(1+3)$-decomposition of the stress-energy tensor, we can write $\mathbb{V}[X]=v+\overline{\mathbb{Q}}+X^{0}$, as follows from (124) and (126).

${ }^{14}$ These gauge choices and others are discussed, for example, by Kodama and Sasaki (1984), Hwang (1991), Hwang and Noh (1999) and Malik and Wands (2009). In contrast to our approach which emphasizes relations between gauge invariants, they define gauge invariants in terms of gauge-variant quantities.
} 
Determining $X^{0}$ in this way does in fact satisfy condition (2), $\Delta X^{0}=\xi^{0}$. This has been verified for $X_{\mathrm{p}}$ and $X_{\mathrm{c}}$ in UW (see equation (26)). For the other two cases, we need the transformation laws:

$$
\Delta^{(1)} T_{0}^{0}=-3 \mathcal{H} \mathcal{M}^{-2} \xi^{0}, \quad \Delta^{(1)} T^{0}{ }_{i}=-\mathcal{M}^{-2} \mathbf{D}_{i} \xi^{0},
$$

which are a consequence of (11) 15 Condition (2) now follows immediately from (24), (25c), (25d) and (26).

In practice, we will not use the explicit expressions for $X^{0}$ that are defined implicitly by equations (25)). Instead, in order to be able to relate gauge invariants associated with different choices of gauge field we introduce a set of transition rules. Let $X_{\bullet}^{0}$ be a specific choice of the temporal gauge field and let $X^{0}$ be an arbitrary choice. The difference

$$
\mathbf{Z}_{\bullet}^{0}[X]:=X_{\bullet}^{0}-X^{0},
$$

is gauge-invariant on account of (21). The desired transition rules are as follows:

$$
\begin{array}{lll}
\Psi[X]=\Psi\left[X_{\bullet}\right]-\mathcal{H} \mathbf{Z}_{\bullet}^{0}[X], & \mathbf{B}[X]=\mathbf{B}\left[X_{\bullet}\right]-\mathbf{Z}_{\bullet}^{0}[X], \\
\mathbb{D}[X]=\mathbb{D}\left[X_{\bullet}\right]-3 \mathcal{H} \mathbf{Z}_{\bullet}^{0}[X], & \mathbb{V}[X]=\mathbb{V}\left[X_{\bullet}\right]-\mathbf{Z}_{\bullet}^{0}[X] .
\end{array}
$$

Equations (28a) follow immediately from (9a), while equations (28b) are a consequence of (19), (21d) and (21e).

By inspection of (28) we see that the following linear combinations of the variables (23) are independent of the choice of $X$ :

$$
\begin{array}{ll}
{[\Psi, \mathbf{B}]:=\Psi[X]-\mathcal{H} \mathbf{B}[X],} & {[\Psi, \mathbb{V}]:=\Psi[X]-\mathcal{H} \mathbb{V}[X],} \\
{[\mathbb{V}, \mathbf{B}]:=\mathbb{V}[X]-\mathbf{B}[X],} & {[\mathbb{D}, \mathbb{V}]:=\mathbb{D}[X]-3 \mathcal{H} \mathbb{V}[X],} \\
{[\mathbb{D}, \mathbf{B}]:=\mathbb{D}[X]-3 \mathcal{H} \mathbf{B}[X],} & {[\mathbb{D}, \Psi]:=\mathbb{D}[X]-3 \Psi[X] .}
\end{array}
$$

We can thus substitute two different choices of $X^{0}$ into any of the $X$-independent expressions in (29) and equate the results, thereby relating different gauge invariants. For example, if we first choose $X^{0}=X_{\mathrm{c}}^{0}$ in $[\mathbb{D}, \Psi]$ and then keep $X^{0}$ arbitrary as the second choice, we obtain

$$
\mathbb{D}\left[X_{\mathrm{c}}\right]=\mathbb{D}[X]-3 \Psi[X],
$$

on account of (25b). If we set $X^{0}=X_{\rho}^{0}$ in this equation it follows that

$$
\mathbb{D}\left[X_{\mathrm{c}}\right]=-3 \Psi\left[X_{\rho}\right],
$$

on account of (25d).

The gauge invariant $\Phi[X]$ is on a different footing from the gauge invariants (23) since it depends on the derivative of $X^{0}$ through equation (9a). Thus requiring $\Phi[X]=0$ does not determine $X^{0}$ uniquely and hence does not lead to a fully fixed gaug 16 . Nevertheless, $\Phi[X]$ does have a well-defined transition rule analogous to (28), namely

$$
\Phi[X]=\Phi\left[X_{\bullet}\right]+\left(\partial_{\eta}+\mathcal{H}\right) \mathbf{Z}_{\bullet}^{0}[X],
$$

\footnotetext{
${ }^{15}$ Note that the formal similarity between (11) and (3) enables one to obtain (26) directly from (19) without any calculation.

${ }^{16}$ As a consequence the synchronous gauge contains residual freedom.
} 
as follows from (9a)). By comparing (32) with (28) one can construct $X$-independent linear combinations of $\Phi[X]$ and the variables in (23)), analogous to (29). For example,

$$
[\Phi, \mathbb{V}]:=\Phi[X]+\left(\partial_{\eta}+\mathcal{H}\right) \mathbb{V}[X],
$$

is independent of $X$. There are three other expressions linking $\Phi[X]$ with $\Psi[X], \mathbf{B}[X]$ and $\mathbb{D}[X]$ that can be written if needed. If we set $X=X_{\mathrm{v}}$ in (33) and use (25c) we obtain

$$
\Phi\left[X_{\mathrm{v}}\right]=\Phi[X]+\left(\partial_{\eta}+\mathcal{H}\right) \mathbb{V}[X],
$$

which we will use later.

To conclude this section we note that the gauge invariants and $X$-independent combinations that we have introduced do not exhaust all possibilities, but do serve to illustrate an efficient way of defining gauge invariants and determining their interrelationships, which constitutes one of the main results of this paper. A further example arises in appendix C.2, where we make use of another gauge invariant, namely the linear perturbation of the Hubble scalar of a timelike reference congruence, denoted by $\mathbf{H}[X]$. In working with this gauge invariant we find it necessary to introduce an $X$-independent combination involving three gauge invariants (see equation (139)).

\section{Notation}

Our general notation for dimensionless gauge invariants is exemplified by $\Psi\left[X_{\mathrm{c}}\right]$ and $\mathbb{V}\left[X_{\mathrm{p}}\right]$, i.e. a capital letter, or a bold face letter, or a special font, e.g. $\mathbb{V}$, which denotes inertial mass-density normalization, replaces the symbol for an associated gauge-variant variable, with the choice of the gauge vector field indicated by a subscript on the symbol $X$. For convenience we will often simplify the notation by setting $\Psi\left[X_{\bullet}\right]=\Psi_{\bullet}$, etc.. For some of the commonly used gauge invariants we will use unsubscripted symbols:

$$
\begin{array}{rll}
\Phi:=\Phi\left[X_{\mathrm{p}}\right], & \Psi:=\Psi\left[X_{\mathrm{p}}\right], & \mathbb{V}:=\mathbb{V}\left[X_{\mathrm{p}}\right], \\
\mathbf{A}:=\Phi\left[X_{\mathrm{c}}\right], & \mathbf{B}:=\mathbf{B}\left[X_{\mathrm{c}}\right], & \mathbb{D}:=\mathbb{D}\left[X_{\mathrm{v}}\right] .
\end{array}
$$

\section{$3 \quad$ Structure of the linearized governing equations}

In this section we give different forms for the governing equations for scalar perturbations, first using the metric gauge invariants as basic variables, and then using the stress-energy gauge invariants.

\subsection{Linearized Einstein field equations}

As shown in UW there are two natural choices of intrinsic metric gauge invariants when formulating the linearized Einstein equations for scalar perturbations, the uniform curvature gauge invariants and the Poisson gauge invariants. As in UW we 
introduce the geometric background scalars $\mathcal{A}_{G}$ and $\mathcal{C}_{G}^{2}$, with $\mathcal{C}_{G}^{2}$ defined in terms of the derivative of $\mathcal{A}_{G}$ :

$$
\mathcal{A}_{G}:=2\left(-\mathcal{H}^{\prime}+\mathcal{H}^{2}+K\right), \quad \mathcal{A}_{G}^{\prime}=-\left(1+3 \mathcal{C}_{G}^{2}\right) \mathcal{H} \mathcal{A}_{G},
$$

(see UW, equation (42)). With $\mathcal{A}_{T}$ and $\mathcal{C}_{T}^{2}$ defined by (16), the background Einstein equations imply that $\mathcal{A}_{G}=\mathcal{A}_{T}$ and $\mathcal{C}_{G}^{2}=\mathcal{C}_{T}^{2}$. We denote their common values by $\mathcal{A}$ and $\mathcal{C}^{2}$ :

$$
\mathcal{A}=\mathcal{A}_{G}=\mathcal{A}_{T}, \quad \mathcal{C}^{2}=\mathcal{C}_{G}^{2}=\mathcal{C}_{T}^{2} .
$$

\section{The uniform curvature formulation}

The governing equations in the uniform curvature formulation are17:

$$
\begin{aligned}
& \mathcal{L}_{B} \mathbf{B}+\mathbf{A}=-\Pi \quad=-\mathcal{A}_{T} \bar{\Pi} \\
& \mathcal{H}\left(\mathcal{L}_{A} \mathbf{A}+\mathcal{C}_{G}^{2} \mathbf{D}^{2} \mathbf{B}\right)=\frac{1}{2} \Gamma+\frac{1}{3} \mathbf{D}^{2} \Pi=\mathcal{A}_{T}\left(\frac{1}{2} \bar{\Gamma}+\frac{1}{3} \mathbf{D}^{2} \bar{\Pi}\right), \\
& \mathcal{H}\left(\mathbf{D}^{2}+3 K\right) \mathbf{B}=-\frac{1}{2} \Delta \quad=-\frac{1}{2} \mathcal{A}_{T} \mathbb{D}, \\
& \mathcal{H} \mathbf{A}+\left(\frac{1}{2} \mathcal{A}_{G}-K\right) \mathbf{B}=-\frac{1}{2} V \quad=-\frac{1}{2} \mathcal{A}_{T} \mathbb{V},
\end{aligned}
$$

where the first order differential operators $\mathcal{L}_{A}$ and $\mathcal{L}_{B}$ are defined by

$$
\mathcal{L}_{A}:=\partial_{\eta}+\mathcal{H B}, \quad \mathcal{L}_{B}:=\partial_{\eta}+2 \mathcal{H},
$$

with

$$
\mathcal{B}:=\frac{2 \mathcal{H}^{\prime}}{\mathcal{H}^{2}}+1+3 \mathcal{C}_{G}^{2} .
$$

For future reference we note that

$$
\mathcal{H B}=-\left(\frac{\mathcal{A}_{G}}{\mathcal{H}^{2}}\right)^{-1}\left(\frac{\mathcal{A}_{G}}{\mathcal{H}^{2}}\right)^{\prime},
$$

as follows from (36).

\section{The Poisson formulation}

The governing equations in the Poisson formulation are (UW, equations (54)):

$$
\begin{aligned}
& \Psi-\Phi=\quad \Pi \quad=\mathcal{A}_{T} \bar{\Pi}, \\
& \left(\mathcal{L}-\mathcal{C}_{G}^{2} \mathbf{D}^{2}\right) \Psi=\frac{1}{2} \Gamma+\left(\frac{1}{3} \mathbf{D}^{2}+\mathcal{H} \mathcal{L}_{A}\right) \Pi=\mathcal{A}_{T}\left(\frac{1}{2} \bar{\Gamma}+\left(\frac{1}{3} \mathbf{D}^{2}+\mathcal{H} \partial_{\eta}+2 \mathcal{H}^{\prime}\right) \bar{\Pi}\right), \\
& \left(\mathbf{D}^{2}+3 K\right) \Psi=\quad \frac{1}{2} \Delta=\frac{1}{2} \mathcal{A}_{T} \mathbb{D}, \\
& \partial_{\eta} \Psi+\mathcal{H} \Phi=\quad-\frac{1}{2} V \quad=-\frac{1}{2} \mathcal{A}_{T} \mathbb{V},
\end{aligned}
$$

where the second order differential operator $\mathcal{L}$ is defined by

$$
\mathcal{L}(\bullet):=\mathcal{H} \mathcal{L}_{A} \mathcal{L}_{B}\left(\frac{\bullet}{\mathcal{H}}\right),
$$

\footnotetext{
${ }^{17}$ See UW, equation (52). We give the matter terms on the right hand side in two forms: using $a$-normalization as in UW, and $\mathcal{M}$-normalization as introduced in the present paper.
} 
or equivalently

$$
\mathcal{L}=\partial_{\eta}^{2}+3\left(1+\mathcal{C}_{G}^{2}\right) \mathcal{H} \partial_{\eta}+\mathcal{H}^{2} \mathcal{B}-\left(1+3 \mathcal{C}_{G}^{2}\right) K
$$

(UW, equation (56)). For a discussion of these two systems of governing equations, and the ways in which they differ, we refer to UW, section 3.2, following equation (56).

\subsection{Linearized conservation equations, without Einstein's field equations}

As shown in Appendix $\mathrm{B}$, linearizing the conservation law $\nabla_{b} T_{a}^{b}=0$ leads to the following gauge-invariant equations:

$$
\begin{aligned}
\partial_{\eta}(\mathbb{D}[X]-3 \Psi[X])+\mathbf{D}^{2}(\mathbb{V}[X]-\mathbf{B}[X]) & =-3 \mathcal{H} \bar{\Gamma} \\
\left(\partial_{\eta}+\mathcal{H}\right) \mathbb{V}[X]+\Phi[X]+\mathcal{C}_{T}^{2} \mathbb{D} & =-\bar{\Gamma}-\bar{\Xi}
\end{aligned}
$$

where

$$
\bar{\Xi}:=\frac{2}{3}\left(\mathbf{D}^{2}+3 K\right) \bar{\Pi},
$$

and $\mathbb{D}[X], \mathbb{V}[X], \bar{\Gamma}$ and $\bar{\Pi}$ are defined by equations (17) and (21).

These equations are valid for any choice of temporal gauge field $X^{0}$. Referring to (29) and (33) we recognize the three groups of terms on the left side as the $X$ independent expressions $[\mathbb{D}, \Psi],[\mathbb{V}, \mathbf{B}]$ and $[\Phi, \mathbb{V}]$ in $(29)$. We choose $X^{0}=X_{\mathrm{c}}^{0}$ and $X^{0}=X_{\mathrm{p}}^{0}$ in the first two, and $X^{0}=X_{\mathrm{v}}^{0}$ in the third, and use (25). Equations (43) then assume the concise form

$$
\begin{aligned}
\partial_{\eta} \mathbb{D}_{\mathrm{c}}+\mathbf{D}^{2} \mathbb{V} & =-3 \mathcal{H} \bar{\Gamma}, \\
\Phi_{\mathrm{v}}+\mathcal{C}_{T}^{2} \mathbb{D} & =-\bar{\Gamma}-\bar{\Xi},
\end{aligned}
$$

where $\mathbb{D}_{\mathrm{c}}=\mathbb{D}\left[X_{\mathrm{c}}\right], \mathbb{V}=\mathbb{V}\left[X_{\mathrm{p}}\right], \mathbb{D}=\mathbb{D}\left[X_{\mathrm{v}}\right]$ and $\Phi_{\mathrm{v}}=\Phi\left[X_{\mathrm{v}}\right]$, in accordance with our convention for labeling gauge invariants. In certain circumstances, the first equation can be interpreted as a conservation law for $\mathbb{D}_{c}$, as will be discussed in section 4 . The second equation 18 shows that for a barotropic perfect fluid $\Phi_{\mathrm{v}}$ is proportional to $\mathbb{D}$, and is in fact zero for dust.

One would like to use equations (43) to obtain a system of evolution equations for the stress-energy gauge invariants $\mathbb{D}[X]$ and $\mathbb{V}[X]$, for some choice of the gauge field $X$. This is not possible due to the presence of the metric variables $\partial_{\eta} \Psi[X]$ and $\Phi[X]$. However, in the case that the stress-energy tensor is the total stressenergy tensor one can use the linearized Einstein equations to eliminate these terms and achieve the desired goal, as we will show in section 3.3. On the other hand equations (43) are valid for each (non-interacting) individual stress-energy tensor of a multi-component source, and as such they form a convenient starting point for the derivation of a simple system of governing equations for scalar perturbations of such a source. We will derive these equations in section 3.4.

\footnotetext{
${ }^{18}$ This equation corresponds to equation (5.20) in Bardeen (1980).
} 


\subsection{Linearized conservation equations in conjunction with Einstein's field equations}

We now use the results of sections 3.1 and 3.2 to derive a system of governing equations in the form of a first order (in time) system of partial differential equations with the stress-energy gauge invariants as basic variables.

Choose $X^{0}=X_{\mathrm{p}}^{0}$ in (43), and eliminate $\mathbb{D}_{\mathrm{p}}$ using $\mathbb{D}=\mathbb{D}_{\mathrm{p}}-3 \mathcal{H} \mathbb{V}$, which is obtained from the $X$-independent invariant $[\mathbb{D}, \mathbb{V}]$ in $(29)$. The resulting equations are

$$
\begin{aligned}
\partial_{\eta}(\mathbb{D}+3 \mathcal{H} \mathbb{V})-3 \partial_{\eta} \Psi+\mathbf{D}^{2} \mathbb{V} & =-3 \mathcal{H} \bar{\Gamma} \\
\left(\partial_{\eta}+\mathcal{H}\right) \mathbb{V}+\Phi+\mathcal{C}_{T}^{2} \mathbb{D} & =-\bar{\Gamma}-\bar{\Xi}
\end{aligned}
$$

using the notation (35). The combination (45a) $-3 \mathcal{H}(45 \mathrm{~b})$ can be rearranged to read

$$
\left(\partial_{\eta}-3 \mathcal{H} \mathcal{C}_{T}^{2}\right) \mathbb{D}+\left(\mathbf{D}^{2}+3 K\right) \mathbb{V}-3\left(\partial_{\eta} \Psi+\mathcal{H} \Phi+\frac{1}{2} \mathcal{A}_{G} \mathbb{V}\right)=3 \mathcal{H} \bar{\Xi}
$$

where $\mathcal{A}_{G}$ is given by (36). If the stress-energy tensor is the total stress-energy tensor, and if we impose Einstein's field equation (40d) and the background field equation $\mathcal{A}_{G}=\mathcal{A}_{T}$, then the above equation simplifies to

$$
\left(\partial_{\eta}-3 \mathcal{H} \mathcal{C}_{T}^{2}\right) \mathbb{D}+\left(\mathbf{D}^{2}+3 K\right) \mathbb{V}=3 \mathcal{H} \Xi .
$$

Equations (47) and (45b) form a coupled first order system of evolution equations for $\mathbb{D}$ and $\mathbb{V}$. However due to the appearance of the metric potential $\Phi$ the system is not closed. We can remedy this deficiency by applying the operator $\mathbf{D}^{2}+3 K$ to (45b) and using

$$
\mathbb{Z}:=\left(\mathbf{D}^{2}+3 K\right) \mathbb{V}
$$

as a new variable to replace $\mathbb{V}$ in the system. On using the Einstein equations (40a) and (40d), which yield

$$
\left(\mathbf{D}^{2}+3 K\right) \Phi=\left(\mathbf{D}^{2}+3 K\right)\left(\Psi-\mathcal{A}_{T} \bar{\Pi}\right)=\frac{1}{2} \mathcal{A}(\mathbb{D}-3 \bar{\Xi})
$$

equations (47), (48) and $\left(\mathbf{D}^{2}+3 K\right)((45 \mathrm{~b}))$ result in

$$
\begin{aligned}
\left(\partial_{\eta}-3 \mathcal{C}_{T}^{2} \mathcal{H}\right) \mathbb{D}+\mathbb{Z} & =3 \mathcal{H} \bar{\Xi} \\
\left(\partial_{\eta}+\mathcal{H}\right) \mathbb{Z}+\left(\frac{1}{2} \mathcal{A}+\mathcal{C}_{T}^{2}\left(\mathbf{D}^{2}+3 K\right)\right) \mathbb{D} & =-\left(\mathbf{D}^{2}+3 K\right)(\bar{\Gamma}+\bar{\Xi})+\frac{3}{2} \mathcal{A} \bar{\Xi},
\end{aligned}
$$

where $\mathcal{A} \equiv \mathcal{A}_{G}=\mathcal{A}_{T}$. For the reader's convenience we note that the variables in these equations are defined by equations (21b), (21c), (43c) and (48).

Equations (50) constitute one of the main results of this paper. They form a coupled system of first order (in time) partial differential equations for $(\mathbb{D}, \mathbb{Z})$, assuming that the stress-energy terms $\bar{\Gamma}$ and $\bar{\Xi}$ are given. They determine the behaviour of the scalar mode of linear perturbations of an FL cosmology with arbitrary stress-energy content. The structure of this system is similar to the structure of the system of evolution equations (38a) and (38b) for the uniform curvature metric gauge invariants $\mathbf{A}$ and $\mathbf{B}$, and can be derived from them as follows. First use (38d) to express $\mathbf{A}$ in terms of $\mathbb{V}$. Then apply the operator $\mathbf{D}^{2}+3 K$ to both equations and use (38c) to express $\left(\mathbf{D}^{2}+3 K\right) \mathbf{B}$ in terms of $\mathcal{A}_{T} \mathbb{D}$, after which some obvious manipulations lead to equations (50). 


\section{The evolution equation for $\mathbb{D}$}

By eliminating $\mathbb{Z}$ from equations (50) one can obtain a second order evolution equation for the gauge-invariant density perturbation $\mathbb{D}$. We apply the operator $\partial_{\eta}+\mathcal{H}$ to the first of equations (50) and use the second equation to eliminate $\mathbb{Z}$. The resulting equation can be written in the form

$$
\left(\mathcal{L}_{\mathcal{D}}-\mathcal{C}^{2} \mathbf{D}^{2}\right) \mathbb{D}=2\left(\mathbf{D}^{2}+3 K\right)\left(\frac{1}{2} \bar{\Gamma}+\left(\frac{1}{3} \mathbf{D}^{2}+\mathcal{H} \partial_{\eta}+2 \mathcal{H}^{\prime}\right) \bar{\Pi}\right)
$$

where

$$
\mathcal{L}_{\mathcal{D}}:=\partial_{\eta}^{2}+\left(1-3 \mathcal{C}^{2}\right) \mathcal{H} \partial_{\eta}+\left(1-3 \mathcal{C}^{2}\right) \mathcal{H}^{\prime}-\left(1+3 \mathcal{C}^{2}\right)\left(\mathcal{H}^{2}+K\right)-3\left(\mathcal{C}^{2}\right)^{\prime} \mathcal{H}
$$

Equation (51a) can also be derived from the governing equations (40) in Poisson form. We apply $\mathbf{D}^{2}+3 K$ to (40b) and use (40c) to relate $\left(\mathbf{D}^{2}+3 K\right) \Psi$ to $\mathbb{D}$. By comparing the resulting evolution equation with (51a), we can conclude that the operator $\mathcal{L}_{\mathcal{D}}$ is related to the operator $\mathcal{L}$ according to

$$
\mathcal{L}(\mathcal{A} \bullet)=\mathcal{A} \mathcal{L}_{\mathcal{D}}(\bullet)
$$

This result can also be verified by direct calculation.

Equation (51a) is a second order linear partial differential equation for $\mathbb{D}$, assuming that the stress-energy terms $\bar{\Gamma}$ and $\bar{\Pi}$ are given. It differs from other related equations in the literature, for example, Ellis et al (1990) (see their equation (48), with the coefficients given by equations (19) and (20)), and Hwang and Noh (1999) (see their equation $(45))$, since we have defined $\mathbb{D}$ by normalizing with $\mathcal{M}^{2}={ }^{(0)} \rho+{ }^{(0)} p$, while the usual practice is to use ${ }^{(0)} \rho$. If the source is a perfect fluid with a linear equation of state and a cosmological constant, i.e.

$$
\rho=\rho_{m}+\Lambda, \quad p=p_{m}-\Lambda, \quad p_{m}=w \rho_{m},
$$

then the right side of (51a) is zero, and one can use Einstein's equations in the background model (UW equations (41)) to write the expression (51b) in the form 19

$$
\mathcal{L}_{\mathcal{D}}=\partial_{\eta}^{2}+(1-3 w) \mathcal{H} \partial_{\eta}-\frac{1}{2}\left[(1+3 w)(1-w) \rho_{m}+4 w \Lambda\right] a^{2} .
$$

In this case (51a) is compatible with those equations cited above.

\subsection{Governing equations for a multi-component source}

The governing equations for a perturbed FL cosmology with a multi-component source were first derived by Kodama and Sasaki (1984), and subsequently considered by various authors including Hwang (1991), Dunsby et al (1992) and Durrer (2008). These authors, as is customary, use normalization with ${ }^{(0)} \rho$ when defining the density perturbation. We have found that the derivation and the form of the governing equations is significantly simpler if one uses $\mathcal{M}$-normalization, as introduced in section 2.2. In this section we thus give a brief derivation of the relevant equations.

\footnotetext{
${ }^{19}$ Since $w=$ constant we have $\mathcal{C}^{2}=w$.
} 
We consider a multi-component source with $n$ separate stress-energy tensors denoted by ${ }_{A} T_{b}^{a}$, with $A=1, \ldots, n$, which sum to form the total stress-energy tensor:

$$
T_{b}^{a}=\sum_{A=1}^{n}{ }_{A}^{a}{ }_{b}
$$

For simplicity we assume that the individual components are non-interacting. As shown in Appendix $\mathrm{B}$, linearizing the conservation equation $\nabla_{b} T_{a}^{b}=0$ for an arbitrary component labeled $A$ leads to the following equation 20 :

$$
\begin{aligned}
& \partial_{\eta}\left(\mathbb{D}_{A}[X]-3 \Psi[X]\right)+\mathbf{D}^{2}\left(\mathbb{V}_{A}[X]-\mathbf{B}[X]\right)=-3 \mathcal{H} \bar{\Gamma}_{A}, \\
&\left(\partial_{\eta}+\mathcal{H}\right) \mathbb{V}_{A}[X]+\Phi[X]+\mathcal{C}_{A}^{2} \mathbb{D}_{A}=-\bar{\Gamma}_{A}-\bar{\Xi}_{A},
\end{aligned}
$$

where

$$
\bar{\Xi}_{A}:=\frac{2}{3}\left(\mathbf{D}^{2}+3 K\right) \bar{\Pi}_{A} .
$$

We assume that the gauge field $X$ does not depend on the labeling index. A quantity $\mathbb{F}_{A}$ associated with ${ }_{A} T_{b}^{a}$ that satisfies

$$
\sum_{A=1}^{n} \mathcal{B}_{A} \mathbb{F}_{A}=\mathbb{F}
$$

where $\mathbb{F}$ is the corresponding quantity associated with $T_{b}^{a}$, will be called additive. Here the coefficients $\mathcal{B}_{A}$ are defined by

$$
\mathcal{B}_{A}:=\frac{\mathcal{A}_{A}}{\mathcal{A}}, \quad \text { and satisfy } \quad \sum_{A=1}^{n} \mathcal{B}_{A}=1
$$

We note that $\mathbb{D}_{A}[X], \mathbb{V}_{A}[X], \mathbb{D}_{A}, \Xi_{A}, \bar{\Gamma}_{A}+\mathcal{C}^{2} \mathbb{D}_{A}[X]$ and $\mathcal{C}_{A}^{2}$ are additive 21$]$.

In order to obtain a closed system of evolution equations we introduce the "difference variables"

$$
\mathbb{D}_{A B}:=\mathbb{D}_{A}[X]-\mathbb{D}_{B}[X], \quad \mathbb{V}_{A B}:=\mathbb{V}_{A}[X]-\mathbb{V}_{B}[X]
$$

It follows from (19a), (19b), (21d) and (21e) that $\mathbb{D}_{A B}$ and $\mathbb{V}_{A B}$ are $X$-independent. In order to obtain evolution equations for $\mathbb{D}_{A B}$ and $\mathbb{V}_{A B}$ we form the difference of two copies of equations (56), labeled $A$ and $B$. In this way we obtain the following equations:

$$
\begin{aligned}
\partial_{\eta} \mathbb{D}_{A B}+\mathbf{D}^{2} \mathbb{V}_{A B} & =-3 \mathcal{H} \bar{\Gamma}_{A B}, \\
\left(\partial_{\eta}+\mathcal{H}\right) \mathbb{V}_{A B}+\mathbb{K}_{A B}+\left(\mathcal{C}_{A}^{2}-\mathcal{C}_{B}^{2}\right) \mathbb{D} & =-\bar{\Gamma}_{A B}-\bar{\Xi}_{A B},
\end{aligned}
$$

\footnotetext{
${ }^{20}$ In these equations $\mathcal{C}_{A}^{2}$ denotes the value of $\mathcal{C}_{T}^{2}$ for the component labeled $A$. We have dropped the subscript $T$ for convenience.

${ }^{21}$ One can check the consistency of (56) by showing that $\sum_{A=1}^{n} \mathcal{B}_{A}(566)=(43)$. Note that $\mathbb{D}_{A}=\mathbb{D}_{A}[X]-3 \mathcal{H} \mathbb{V}_{A}[X]$.

${ }^{22}$ The only calculation involves showing that $\mathcal{C}_{A}^{2} \mathbb{D}_{A}-\mathcal{C}_{B}^{2} \mathbb{D}_{B}=\mathbb{K}_{A B}+\left(\mathcal{C}_{A}^{2}-\mathcal{C}_{B}^{2}\right) \mathbb{D}$, which follows by writing $\mathbb{D}_{A}=\mathbb{D}+\sum_{C=1}^{n} \mathcal{B}_{C}\left(\mathbb{D}_{A}-\mathbb{D}_{C}\right)$, and a similar expression for $\mathbb{D}_{B}$.
} 
where

$$
\begin{aligned}
\mathbb{K}_{A B} & :=\sum_{C=1}^{n} \mathcal{B}_{C}\left(\mathcal{C}_{A}^{2}\left(\mathbb{D}_{A C}-3 \mathcal{H} \mathbb{V}_{A C}\right)-\mathcal{C}_{B}^{2}\left(\mathbb{D}_{B C}-3 \mathcal{H} \mathbb{V}_{B C}\right)\right), \\
\bar{\Gamma}_{A B} & :=\bar{\Gamma}_{A}-\bar{\Gamma}_{B}, \quad \bar{\Xi}_{A B}:=\bar{\Xi}_{A}-\bar{\Xi}_{B},
\end{aligned}
$$

Equations (61) do not form a closed system for $\mathbb{D}_{A B}$ and $\mathbb{V}_{A B}$, however, due to the appearance of the total intrinsic matter gauge invariant $\mathbb{D}$. The evolution equation (51a) for $\mathbb{D}$ contains $\bar{\Gamma}$ and $\bar{\Xi}$ as source term 23 , which have to be expressed in terms of $\bar{\Gamma}_{A}, \bar{\Xi}_{A}$ and $\mathbb{D}_{A B}$. The term $\bar{\Gamma}$ is not additive, whereas $\bar{\Gamma}+\mathcal{C}^{2} \mathbb{D}[X]$ is, i.e.

$$
\bar{\Gamma}+\mathcal{C}^{2} \mathbb{D}[X]=\sum_{A=1}^{n} \mathcal{B}_{A}\left(\bar{\Gamma}_{A}+\mathcal{C}_{A}^{2} \mathbb{D}_{A}[X]\right) .
$$

It follows that $\bar{\Gamma}$ can be expressed 24 as a sum involving $\bar{\Gamma}_{A}$ and $\mathbb{D}_{A B}$ :

$$
\bar{\Gamma}=\sum_{A=1}^{n} \mathcal{B}_{A} \bar{\Gamma}_{A}+\frac{1}{2} \sum_{A, B=1}^{n} \mathcal{B}_{A} \mathcal{B}_{B}\left(\mathcal{C}_{A}^{2}-\mathcal{C}_{B}^{2}\right) \mathbb{D}_{A B}
$$

On the other hand, the term $\Xi$ is additive:

$$
\bar{\Xi}=\sum_{A=1}^{n} \mathcal{B}_{A} \bar{\Xi}_{A}
$$

In conclusion, equations (61) and the evolution equation (51a) for $\mathbb{D}$, in conjunction with equations (63), form a closed system for $\mathbb{D}_{A B}, \mathbb{V}_{A B}$ and $\mathbb{D}$ that describes the scalar mode of a perturbed FL cosmology with a multi-component source 25 . We note that all gauge invariants in these equations are $X$-independent.

\subsection{Gauge-fixing versus gauge-invariance}

To conclude this section we comment briefly on the two points of view as regards formulating the governing equations for scalar perturbations. In the gauge-fixing approach one chooses a gauge $a b$ initio, in which case all the gauge invariants that appear in the governing equations are associated with this particular gauge. Recent references that use this traditional approach are Mukhanov (2005) and Weinberg (2008). In contrast, one can work with a variety of gauge invariants in the spirit of Bardeen (1980), in which case one has the flexibility to use gauge invariants associated with different gauges in formulating the governing equations. A notable

\footnotetext{
${ }^{23}$ Note that the term $\left(\mathbf{D}^{2}+3 K\right) \bar{\Pi}$ on the right side of (51a) can be replaced by $\bar{\Xi}$ on account of (43c).

${ }^{24}$ Substitute $\mathbb{D}_{A}[X]=\mathbb{D}[X]+\sum_{C=1}^{n} \mathcal{B}_{C} \mathbb{D}_{A C}$ in $(\underline{62})$ and use $\sum_{A=1}^{n} \mathcal{B}_{A} \mathcal{C}_{A}^{2}=\mathcal{C}^{2}$

${ }^{25}$ For the reader's convenience we give the equation numbers in the previously mentioned references that correspond to our equations (61): Kodama and Sasaki (1984), (5.59)-(5.60), Hwang (1991), (37)-(38), Bruni et al (1992b), (91)-(92), Dunsby et al (1992), (86)-(87), and Durrer (2008), (2.136)-(2.137).
} 
example is the generalized Poisson equation (40C), which relates the Bardeen potential $\Psi \equiv \Psi\left[X_{\mathrm{p}}\right]$ to the matter density gauge invariant $\mathbb{D} \equiv \mathbb{D}\left[X_{\mathrm{v}}\right]$. A variety of other examples occur in this paper, including the system of equations (50) for $\mathbb{D}_{\mathrm{v}}$ and $\mathbb{Z}_{\mathrm{p}}$, equations (38c) and (38d) in the uniform curvature formulation, and the conservation law (66) .

\section{Conserved quantities}

Two "conserved quantities" that are associated with scalar perturbations of FL have been defined in the literature. These quantities, often denoted by $\zeta$, satisfy an evolution equation of the form

$$
\partial_{\eta} \zeta_{\bullet}=\mathbf{D}^{2} \mathbf{C} \bullet+\mathcal{H} \bar{\Gamma},
$$

where $\mathbf{C}$. is an expression involving the primary gauge invariants such as $\Psi$ or $\mathbb{V}$ and the background variables. This equation is referred to as a "conservation law", since if spatial derivatives are negligible ("perturbations outside the horizon") and if $\bar{\Gamma}$ is zero or negligible in some epoch, then (64) is approximated by $\partial_{\eta} \zeta \bullet=0$, i.e. $\zeta \bullet$ is approximately constant in time during that epoch.

Two of the evolution equations that we have presented in section 3. namely equations (44a) and (38b), can be written in the form (64) simply by multiplying by a suitable factor. The conserved quantity can then be identified by inspection. We consider each in turn.

\section{The conserved quantity $\zeta_{\rho}$}

The evolution equation (44a) for $\mathbb{D}_{\mathrm{c}}$ has the form of a conservation law (64). We multiply by the numerical factor $-\frac{1}{3}$ to agree with current convention, and comparison with (64) leads to the following definition of the first conserved quantity:

$$
\zeta_{\rho}:=-\frac{1}{3} \mathbb{D}_{\mathrm{c}} .
$$

Equation (44a) assumes the form

$$
\partial_{\eta} \zeta_{\rho}=\frac{1}{3} \mathbf{D}^{2} \mathbb{V}+\mathcal{H} \bar{\Gamma} .
$$

Our motivation for using the notation $\zeta_{\rho}$ is that on account of equation (31) we have

$$
\zeta_{\rho}=\Psi_{\rho} .
$$

This conservation law was apparently first given in a form closely related to the above by Wands et al (2000) ${ }^{26}$ who emphasized that it depends only on the conservation equation for the stress-energy tensor, i.e. it is independent of Einstein's equations. They denoted our $\zeta_{\rho}$ by $\zeta$ and because of (67) they referred to it as "the

\footnotetext{
${ }^{26}$ See their equations (8) and (9). Their evolution equation (18) corresponds to our equation (66), although their equation is not in a manifestly gauge-invariant form, and uses clock time rather than conformal time. See also Malik and Wands (2009), equations (7.61), (7.62) and (8.35), which are somewhat closer in form to our equations.
} 
curvature perturbation on uniform density surfaces." The quantity $\zeta_{\rho}$ has its origins in the paper Bardeen, Steinhardt and Turner (1983), and was further studied from a different point of view by Brandenberger and Khan (1984)27.

A major step in understanding the significance of $\zeta_{\rho}$ was taken by Langlois and Vernizzi (2005). Motivated by the $1+3$ covariant approach, they showed that this quantity could be obtained as the linearization of an exact nonlinear evolution equation for a certain covector. This approach enabled them to extend the definition of $\zeta_{\rho}$ to second-order (and higher order) perturbations. We refer to their equations (20) and (25) for the general situation and note that their equations (41) and (42) correspond to our equations (67) and (66)

\section{The conserved quantity $\zeta_{\mathrm{v}}$}

The second conservation equation arises from the evolution equation (38b) for $\mathbf{A} \equiv$ $\Phi_{\text {c }}$. Using (39c) we can write the differential operator $\mathcal{L}_{A}$ in the form:

$$
\mathcal{H} \mathcal{L}_{A}(\bullet)=\frac{\mathcal{A}_{G}}{\mathcal{H}} \partial_{\eta}\left(\frac{\mathcal{H}^{2}}{\mathcal{A}_{G}} \bullet\right)
$$

Thus on multiplying (38b) by $2 \mathcal{H} / \mathcal{A}_{G}$ we can write it in the form of a conservation law 28

$$
\partial_{\eta}\left(\frac{2 \mathcal{H}^{2}}{\mathcal{A}} \Phi_{\mathrm{c}}\right)=2 \mathcal{H} \mathbf{D}^{2}\left(\mathcal{C}^{2} \frac{\Psi}{\mathcal{A}}+\frac{1}{3} \bar{\Pi}\right)+\mathcal{H} \bar{\Gamma}
$$

where we have chosen to replace $\mathbf{B}$ by $\Psi$, using the relation $\Psi=-\mathcal{H} \mathbf{B}$. Comparing (69) with (64) leads to the following definition of the second conserved quantity:

$$
\zeta_{\mathrm{v}}:=\frac{2 \mathcal{H}^{2}}{\mathcal{A}} \Phi_{\mathrm{c}}
$$

Equation (69), which is equivalent to (38b), is the conservation equation for $\zeta_{\mathrm{v}}$. An immediate consequence of this equation is that if the source is pressure-free matter plus possibly a cosmological constant, then $\zeta_{\mathrm{v}}$ is constant in time.

We can derive an alternate expression for the conserved quantity $\zeta_{\mathrm{v}}$ as follows. Using the relation $\Psi=-\mathcal{H} \mathbf{B}$ and the definition (70), the velocity equation (38d) can be written in the form:

$$
\zeta_{\mathrm{v}}=\left(1-\frac{2 K}{\mathcal{A}}\right) \Psi-\mathcal{H} \mathbb{V}
$$

We use the $X$-independent gauge invariant $[\Psi, \mathbb{V}]$ in $(29)$ to obtain

$$
\Psi_{\mathrm{v}}=\Psi-\mathcal{H} \mathbb{V}
$$

\footnotetext{
${ }^{27}$ It is not immediately obvious that the expressions given in these papers (equations (2.43) and (2.45) in Bardeen et al (1983) and equations (2.11) and (2.12) in Brandenberger and Khan (1984)) agree with our expressions.

${ }^{28}$ Throughout the remaining discussion about conserved quantities we use the background Einstein equations and hence $\mathcal{A}_{G}=\mathcal{A}_{T}=\mathcal{A}$ and $\mathcal{C}_{G}^{2}=\mathcal{C}_{T}^{2}=\mathcal{C}^{2}$.
} 
which when inserted in (171) leads to

$$
\zeta_{\mathrm{v}}=\Psi_{\mathrm{v}}-\frac{2 K}{\mathcal{A}} \Psi
$$

The quantity $\zeta_{\mathrm{v}}$ is most commonly used when the background curvature is zero $(K=0)$ in which case

$$
\left.\zeta_{\mathrm{v}}\right|_{K=0}=\Psi_{\mathrm{v}} .
$$

This expression motivates our use of the notation $\zeta_{\mathrm{v}}$. Malik and Wands (2009) use the notation $\mathcal{R}$ for $\zeta_{\mathrm{v}}$ in the case $K=0$ (see equation (7.46)), and refer to it as "the curvature perturbation in the comoving gauge." This quantity has its origin in the paper Bardeen (1980) (see equations (5.19) and (5.21)).

There is another commonly used expression for $\zeta_{\mathrm{v}}$, in terms of the Bardeen potential $\Psi$ and its time derivative, which we can quickly derive. On solving (38a) for $\mathbf{A} \equiv \Phi_{\mathrm{c}}$, the definition (70) yields

$$
\zeta_{\mathrm{v}}=\frac{2 \mathcal{H}^{2}}{\mathcal{A}_{G}}\left(\mathcal{L}_{B}\left(\frac{\Psi}{\mathcal{H}}\right)-\Pi\right),
$$

where we used $\mathcal{H} \mathbf{B}=-\Psi$ and where $\mathcal{L}_{B}$ is defined by (39a). We expand the operator and use (36) to express $\mathcal{H}^{\prime}$ in terms of $\mathcal{A}_{G}$. On specializing to flat FL (i.e. $K=0$ ) and setting $\Pi=0$ we obtain the expression

$$
\left.\zeta_{\mathrm{v}}\right|_{K=0}=\Psi+\frac{2}{3(1+w)}\left(\frac{1}{\mathcal{H}} \Psi^{\prime}+\Psi\right),
$$

where $w:={ }^{(0)} p /{ }^{(0)} \rho$. Here we have used the background field equation $\mathcal{A}_{G}=\mathcal{A}_{T}$, and the fact that $\mathcal{A}_{T}=3(1+w) \mathcal{H}^{2}$ when $K=0$, as follows from UW (see equations (41a) and (42a)). The familiar expression (76) can be found, for example, in Mukhanov et al (1992), equation (5.23), and Mukhanov (2005), equation (7.73).

We conclude this section by showing that $\zeta_{\rho}$ and $\zeta_{\mathrm{v}}$, despite their different origins, are closely related. On account of (67) and (73)

$$
\zeta_{\rho}-\zeta_{\mathrm{v}}=\Psi_{\rho}-\Psi_{\mathrm{v}}+\frac{2 K}{\mathcal{A}} \Psi=-\frac{1}{3} \mathbb{D}+\frac{2 K}{\mathcal{A}} \Psi
$$

where we have used the $X$-independent invariant $[\mathbb{D}, \Psi]$ in $(29)$ to obtain the second equality. The generalized Poisson equation (40c) can be used to eliminate $\mathbb{D}$ yielding

$$
\zeta_{\rho}-\zeta_{\mathrm{v}}=-\frac{2}{3 \mathcal{A}} \mathbf{D}^{2} \Psi
$$

This equation suggests that if spatial derivatives are negligible in some epoch, then $\zeta_{\rho} \approx \zeta_{\mathrm{v}}$ in that epoch.

\section{A coupled system for $\left(\zeta_{\mathrm{v}}, \Psi\right)$}

The conservation equation (69) for $\zeta_{\mathrm{v}}$ is one of the linearized Einstein equations in uniform curvature form, namely, the evolution equation for $\mathbf{A}=\Phi_{\mathrm{c}}$. It is helpful to 
also write the evolution equation (38a) for $\mathbf{B}$ in terms of $\zeta_{\mathrm{v}}$, replacing $\mathbf{B}$ by $\Psi$ using the relation $\Psi=-\mathcal{H B}$. The resulting pair of equations has the following form:

$$
\begin{aligned}
\partial_{\eta}\left(\frac{x^{2}}{\mathcal{H}} \Psi\right)-\mathcal{F} \zeta_{\mathrm{v}} & =x^{2} \mathcal{A} \bar{\Pi}, \\
\partial_{\eta} \zeta_{\mathrm{v}}-\frac{\mathcal{C}^{2}}{\mathcal{F}} \mathbf{D}^{2}\left(\frac{x^{2}}{\mathcal{H}} \Psi\right) & =\mathcal{H}\left(\bar{\Gamma}+\frac{2}{3} \mathbf{D}^{2} \bar{\Pi}\right),
\end{aligned}
$$

where $x:=a / a_{*}$ is the dimensionless scale factor, with $a_{*}$ being the scale factor at some reference time, and

$$
\mathcal{F}:=\frac{x^{2} \mathcal{A}}{2 \mathcal{H}^{2}} \text {. }
$$

The system of equations (79) is a particularly useful form of the governing equations for scalar perturbations of FL. The second equation is the "conservation equation" for $\zeta_{\mathrm{v}}$, while the first equation enables one to express the Bardeen potential $\Psi$ as a quadrature, in situations in which $\zeta_{\mathrm{v}}$ is a temporal constant (or can be treated as such) and $\bar{\Pi}$ is negligible:

$$
\Psi\left(\eta, x^{i}\right)=\frac{\mathcal{H}}{x^{2}}\left(\zeta_{\mathrm{v}}\left(x^{i}\right) \int_{0}^{\eta} \mathcal{F} d \bar{\eta}+C_{-}\left(x^{i}\right)\right) .
$$

In particular this equation gives the exact solution where the source is pressure-free matter and, possibly, a cosmological constant (since then $\zeta_{\mathrm{v}}$ is a temporal constant), and the approximate solution in the long wavelength limit, in both cases without restriction on the background spatial curvature $K$.

\section{The $1+3$ gauge-invariant approach}

In this section we first give a concise derivation of the governing equations for linear scalar perturbations in the $1+3$ approach, combining the formulation of Bruni, Dunsby and Ellis (1992a) (hereafter referred to as BDE) 29 with our overall strategy of creating dimensionless quantities by normalizing with $\mathcal{M}$ and $a$. We then introduce the dependence of the $1+3$ variables and differential operators on a perturbation parameter $\epsilon$, which enables us to relate the variables and governing equations of the two approaches in a precise manner. In this way we set the stage for extending the $1+3$ approach to second order.

The $1+3$ gauge-invariant approach to cosmological perturbations is based on choosing a preferred unit timelike vector field $u^{a}$ and decomposing the stress-energy tensor relative to this vector field:

$$
T_{b}^{a}=(\rho+p) u^{a} u_{b}+p \delta_{b}^{a}+\left(q^{a} u_{b}+u^{a} q_{b}\right)+\pi_{b}^{a},
$$

where

$$
u^{a} q_{b}=0, \quad \pi_{a}^{a}=0, \quad u_{a} \pi_{b}^{a}=0 .
$$

\footnotetext{
${ }^{29} \mathrm{BDE}$ give a comprehensive account of the linearization of the full system of equations in the $1+3$ formalism (Ricci and Bianchi identities, and stress-energy conservation equations ). We are concerned only with a limited subset of these equations.
} 
One distinguishes between physical and geometrical quantities which are non-zero in the background spacetime, namely $\rho, p$ and the Hubble scalar $H$ associated with $u^{a}$, and quantities which are zero in the background, such as the stress-energy quantities $q_{a}, \pi_{b}^{a}$, the shear of the preferred congruence, the Weyl curvature tensor and the spatial gradients of $\rho, p$ and $H$ orthogonal to $u^{a}$. The $1+3$ approach focusses on the latter quantities, which are gauge-invariant on account of the Stewart-Walker Lemma.

\subsection{Evolution equations}

The spatial gradients of $\rho$ and $H$ describe the perturbation in a gauge-invariant way at the linear level. However, in order to extract the scalar mode of the perturbation it is necessary to form scalar quantities. We thus take the spatial divergence of these spatial gradients and form the dimensionless spatial Laplacian:

$$
D:=\left(a^{2(3)} \nabla^{2}\right) \mathcal{M}^{2} \rho, \quad Z:=3\left(a^{2(3)} \nabla^{2}\right) a H,
$$

where

$$
{ }^{(3)} \nabla_{a}:=h_{a}^{b} \nabla_{b}, \quad{ }^{(3)} \nabla^{2}:=g^{a b(3)} \nabla_{a}^{(3)} \nabla_{b},
$$

and

$$
h_{a}^{b}:=\delta_{a}^{b}+u_{a} u^{b} .
$$

Note that in introducing dimensionless variables we are normalizing the energy density $\rho$ with $\mathcal{M}^{2}$ and the geometric quantity $H$ with the background scale factor $a$. Likewise we normalize the geometric operator ${ }^{(3)} \nabla^{2}$ with $a^{2}$.

The governing equations for the scalar mode take the form of a coupled system of first order (in time) partial differential equations for $D$ and $Z$. These equations arise from the energy conservation equation (the exact evolution equation for $\rho$ ) and the Raychaudhuri equation (the exact evolution equation for $H$ ). To derive the governing equations one simply applies the differential operator $a^{2(3)} \nabla^{2}$ to the linearized versions of the evolution equations for $\rho$ and $H$, which are obtained by dropping products of first order quantites.

The linearized evolution equations for $D$ and $Z$, derived in Appendix C, are as follow: 30 :

$\begin{aligned} D^{\prime}-3 \mathcal{H} \mathcal{C}_{T}^{2} D+Z & =3 \mathcal{H}(\tilde{\Pi}+\tilde{\Upsilon})-\tilde{\mathbf{D}}^{2} \tilde{Q} \\ Z^{\prime}+\mathcal{H} Z+\left[\frac{1}{2} \mathcal{A}+\mathcal{C}_{T}^{2}\left(\tilde{\mathbf{D}}^{2}+3 K\right)\right] D & =-\left(\tilde{\mathbf{D}}^{2}+3 K\right)(\tilde{\Gamma}+\tilde{\Pi}+\tilde{\Upsilon})+\frac{3}{2} \mathcal{A}(\tilde{\Pi}+\tilde{\Upsilon}),\end{aligned}$

In these equations the dimensionless operators ' and $\tilde{\mathbf{D}}^{2}$ are defined by

$$
A^{\prime}:=a u^{a} \nabla_{a} A, \quad \tilde{\mathbf{D}}^{2} A:=a^{2(3)} \nabla^{2} A,
$$

\footnotetext{
${ }^{30}$ Equations equivalent to (86) have been derived by Woszczyna and Kulak (1989) in the case of a barotropic perfect fluid, using the method of Appendix Cl but with different normalization factors (see their equations (11) and (18)). Their variables $\Delta \epsilon$ and $\Delta \theta$ are related to $D$ and $Z$ according to $D=a^{2} \mathcal{M}^{2} \Delta \epsilon, \quad Z=a^{3} \Delta \theta$.
} 
where $A$ is a scalar. The source terms $\tilde{\Pi}, \tilde{Q}$ and $\tilde{\Upsilon}$ are first order dimensionless scalars formed by taking the spatial divergence of $q^{a}$ and $\pi_{b}^{a}$ after normalizing with $\mathcal{M}^{2}$ :

$$
\tilde{Q}:=\tilde{\mathbf{D}}^{a}\left(\mathcal{M}^{2} q_{a}\right), \quad \tilde{\Pi}:=\tilde{\mathbf{D}}_{a} \tilde{\mathbf{D}}^{b}\left(\mathcal{M}^{2} \pi_{b}^{a}\right), \quad \tilde{\Upsilon}:=\tilde{Q}^{\prime}-\left(3 \mathcal{C}_{T}^{2}-1\right) \mathcal{H} \tilde{Q},
$$

where

$$
\tilde{\mathbf{D}}_{a}:=a^{(3)} \nabla_{a} .
$$

The entropy perturbation $\tilde{\Gamma}$ is given by 31

$$
\tilde{\Gamma}=P-\mathcal{C}_{T}^{2} D,
$$

where

$$
P:=\tilde{\mathbf{D}}^{2}\left(\mathcal{M}^{2} p\right) .
$$

We conclude this section by relating our approach to that of $\mathrm{BDE} 32$. The variables $D$ and $Z$ differ from those introduced by BDE as regards the normalization of $\rho_{m}$ and $H$. Specifically, BDE define

$$
\Delta:=a^{(3)} \nabla^{a}\left(\frac{a^{(3)} \nabla_{a} \rho_{m}}{\rho_{m}}\right), \quad \mathcal{Z}:=3 a^{(3)} \nabla^{a}\left(a^{(3)} \nabla_{a} H\right) .
$$

At the linear level, the factor $\rho_{m}$ in the denominator can be replaced by ${ }^{(0)} \rho_{m}$. In the $1+3$ approach the scale factor is usually defined using the Hubble scalar $H$ of the preferred congruence $u^{a}$, according to $\left(u^{a} \nabla_{a} a\right) / a=H$, in which case ${ }^{(3)} \nabla^{a} a \neq 0$. At the linear level, however, the factor of $a$ can be taken outside ${ }^{(3)} \nabla^{a}$, since the term ${ }^{(3)} \nabla^{a} a$ appears as a product with ${ }^{(3)} \nabla^{a} \rho_{m}$ or ${ }^{(3)} \nabla_{a} H$, and hence can be dropped. To avoid this complication we have chosen the scale factor $a$ to be the scale factor in the background model so that ${ }^{(3)} \nabla^{a} a=0$. This choice also facilitates the link with the metric-based approach. In view of these remarks, the BDE variables (92) can be written in the form:

$$
\Delta=\frac{1}{{ }^{(0)} \rho_{m}} \tilde{\mathbf{D}}^{2} \rho_{m}, \quad \mathcal{Z}=3 \tilde{\mathbf{D}}^{2} H,
$$

which implies that the BDE variables are related to ours according tt 33

$$
\Delta=(1+w) D, \quad a \mathcal{Z}=Z,
$$

since $1 / \mathcal{M}^{2}=(1+w)^{(0)} \rho_{m}$. Our evolution equations (866) for $D$ and $Z$ are equivalent to equations (68) and (69) for $\Delta$ and $\mathcal{Z}$ in $\mathrm{BDE}$, but are simpler in form due to our use of dimensionless variables, in particular our use of $\mathcal{M}$-normalization 34 .

\footnotetext{
${ }^{31}$ See BDE equations (27), (28) and (40). Note that $\tilde{\Gamma}=\frac{w}{1+w} \mathcal{E}$.

${ }^{32} \mathrm{BDE}$ do not incorporate the cosmological constant into the stress-energy tensor as we do. In making a comparison we have to write $\rho=\rho_{m}+\Lambda, p=p_{m}-\Lambda$, with $w=p_{m} / \rho_{m}$ not necessarily constant.

${ }^{33}$ Note that $\rho+p=\rho_{m}+p_{m}=(1+w) \rho_{m}$, and that $\tilde{\mathbf{D}}^{2} \rho_{m}=\tilde{\mathbf{D}}^{2} \rho$.

${ }^{34}$ Our variables $(\tilde{Q}, \tilde{\Pi}, \tilde{\Upsilon})$ are related to those used in the above reference according to $\Psi_{B D E}=\tilde{Q}, \quad a \Pi_{B D E}=\tilde{\Pi}, \quad a F_{B D E}=\tilde{\Upsilon}$.
} 


\subsection{Relation with the metric-based approach}

Equations (86) are closely related to the governing equations in the form (50) for the variables $\mathbb{D}$ and $\mathbb{Z}$, that arise in the metric-based approach. Indeed a formal similarity is obvious on inspection. However, in order to relate the two sets of equations we have to regard each of the variables $D, Z, \tilde{Q}, \tilde{\Pi}$ and $\tilde{\Gamma}$ in (86) as being a function of the perturbation parameter $\epsilon$, which can be expanded in a power series of the form:

$$
F(\epsilon)={ }^{(0)} F+\epsilon^{(1)} F+\ldots .
$$

Since each variable is zero in the background we have ${ }^{(0)} F=0$, while ${ }^{(1)} F$ is the linear perturbation of $F$. We also need to consider the dependence of the differential operators on $\epsilon$, which is as follows:

$$
\left(A^{\prime}\right)(\epsilon)=a u^{a}(\epsilon)^{\epsilon} \nabla_{a} A(\epsilon), \quad\left(\tilde{\mathbf{D}}^{2} A\right)(\epsilon)=a^{2} h^{a b}(\epsilon)^{\epsilon} \boldsymbol{\nabla}_{a}{ }^{\epsilon} \nabla_{b} A(\epsilon)
$$

Assuming that $A$ is a scalar such that ${ }^{(0)} A=0$, it follows that

$$
{ }^{(1)}\left(A^{\prime}\right)=\partial_{\eta}{ }^{(1)} A, \quad{ }^{(1)}\left(\tilde{\mathbf{D}}^{2} A\right)=\mathbf{D}^{2}{ }^{(1)} A,
$$

as is shown in Appendix C.

If we now differentiate equations (86) with respect to $\epsilon$ and set $\epsilon=0$ the resulting equations have precisely the same form but with each variable replaced by its linear perturbation and each differential operator replaced by the corresponding zeroth order operator. We finally have to do a calculation using (95) to specifically relate the variables in the two sets of equations (86) and (50). The details are given in Appendix C, where it is shown that:

$$
\begin{aligned}
& \mathbf{D}^{2} \mathbb{D}={ }^{(1)} D-3 \mathcal{H}^{(1)} \tilde{Q}, \\
& \mathbf{D}^{2} \mathbb{Z}={ }^{(1)} Z+\left(\mathbf{D}^{2}+3 K-\frac{3}{2} \mathcal{A}_{G}\right)^{(1)} \tilde{Q} \\
& \mathbf{D}^{2} \bar{\Xi}={ }^{(1)} \tilde{\Pi}, \\
& \mathbf{D}^{2} \bar{\Gamma}={ }^{(1)} \tilde{\Gamma} .
\end{aligned}
$$

If we now apply the operator $\mathbf{D}^{2}$ to equations (50) and use equations (98) then we obtain precisely equations (86), with each variable by its linear perturbation and each differential operator replaced by its zeroth order perturbation.

\section{Discussion}

In this paper we have presented an efficient way of defining dimensionless gauge invariants and determining their inter-relationships, which we have applied to give a unified account of the various ways of formulating the governing equations for scalar perturbations of FL cosmologies. In defining gauge invariants we use our version of Nakamura's geometrical method, as described in UW (see section 2.1) which is based on specifying a so-called gauge field $X$, and normalizing so as to obtain dimensionless quantities. It turns out that the choice of the spatial part $X^{i}$ of the gauge field does not affect the form of the governing equations for linear perturbations given in UW 
and in the present paper 35 In these papers, however, we find it convenient to fix the spatial part $X^{i}$ of the gauge field according to (7), which leads to a simple form (8) for the metric gauge invariant $\mathbf{f}_{a b}[X]$. This in turn shortens the calculation of the Riemann gauge invariants in UW (see (B.23)) and of the gauge-invariant form of the divergence of the stress-energy tensor in the present paper, equation (43) 36 The remaining gauge freedom is then described by the temporal part $X^{0}$, which we specify uniquely by requiring that one of the four basic gauge invariants $\Psi[X], \mathbf{B}[X], \mathbb{D}[X]$ and $\mathbb{V}[X]$ be zero. This approach eliminates the need to express $X^{0}$ explicitly in terms of gauge-variant variables, and in particular enables one to perform a change of gauge without using $X^{0}$, as in subsection 2.3. Indeed, although the gauge field $X^{a}$, which is gauge-variant, plays an important role in establishing our formalism, we do not use it in performing calculations, in keeping with our goal of working exclusively with gauge invariants. Because it simplifies calculations this approach will facilitate the extension to second order perturbations.

The coupled system of first order partial differential equations for $\mathbb{D}$ and $\mathbb{Z}$, given by equations (50), plays a central role in this paper. To the best of our knowledge they have not been given in the literature. They arise first of all from the linearized conservation equations in conjunction with the linearized Einstein equations, but can also be derived directly from the latter equations, when they are written in terms of the uniform curvature gauge invariants as in (38). Further, as shown in section 5 these equations are essentially equivalent to the first order governing equations for $D$ and $Z$ that arise in the $1+3$ gauge-invariant approach (see equations (86)).

Our derivation of the expressions for the conserved quantities $\zeta_{\rho}$ and $\zeta_{\mathrm{v}}$ deserves comment. We have shown that the conservation equations for $\zeta_{\rho}$ and $\zeta_{\mathrm{v}}$ are simply two of the first order governing equations for scalar perturbations when they are written in terms of the appropriate gauge invariants: the gauge-invariant expression for $\zeta_{\rho}$ arises directly from the linearized conservation equations for the stress-energy tensor, while that for $\zeta_{\mathrm{v}}$ arises from the linearized Einstein equations for the uniform curvature metric gauge invariants. Other expressions for the conserved quantities are derived using the method for finding inter-relationships between gauge invariants given in section 2.3. We mention that $\zeta_{\mathrm{v}}$ is usually introduced by rewriting the second order evolution equation (40b) for the Bardeen potential $\Psi$ in a first order form, a procedure that involves a tedious calculation 37 Our use of the uniform curvature metric gauge invariants avoids the need for any calculation.

Our discussion of the $1+3$ gauge-invariant approach has several novel features. An advantage of this approach is that it is coordinate-free, so that calculations require only standard operations from differential geometry. This feature has enabled us to give a particularly concise derivation of the first order system of governing

\footnotetext{
${ }^{35}$ This feature of linear perturbations is due to the fact that the components of the perturbed Riemann tensor and of the perturbed stress-energy tensor that are used in deriving the governing equations are invariant under spatial gauge transformations. Indeed, according to Bardeen (1988), since the background 3-space is homogeneous and isotropic the perturbations in all physical quantities must be invariant under spatial gauge transformations. Whether this property holds for nonlinear perturbations requires further investigation.

${ }^{36}$ If one does not fix $X^{i}$ one has to use the general form (11) of $\mathbf{f}_{a b}[X]$. However, during the calculations the terms involving $X^{i}$ cancel, leading to the same final results.

${ }^{37}$ This calculation is much easier if one uses our factorization property (41) for the operator $\mathcal{L}$.
} 
equations for scalar perturbations, as given by equations (86) (see Appendix C) 38 We have also derived the relation between the variables $(\mathbb{D}, \mathbb{Z})$ in the metric-based approach and the $1+3$ variables $(D, Z)$ (see equation (98)). A drawback of the $1+3$ approach is that the linearization process is conceptually less clear than in the metric-based approach, relying as it does on "dropping products of first order terms". In relating the $1+3$ approach to the metric-based approach it was necessary to regard $D, Z$ and the differential operators as functions of the perturbation parameter $\epsilon$ and explicitly calculate their dependence on $\epsilon$ to linear order. Introducing the perturbation parameter clarifies the linearization process and points the way to extending the $1+3$ approach to second order perturbations.

\section{Acknowledgments}

CU is supported by the Swedish Research Council (VR grant 621-2009-4163). CU also thanks the Department of Applied Mathematics at the University of Waterloo for kind hospitality. JW acknowledges financial support from the University of Waterloo.

\section{A The Replacement Principle}

We define

$$
I_{a}(\epsilon):=\mathcal{M}^{2 \epsilon} \nabla_{b} T_{a}^{b}(\epsilon) .
$$

The linear perturbation of $I_{a}$, given in equation (110), can be written symbolically in the form

$$
{ }^{(1)} I_{a}=\mathrm{L}_{a}\left(\mathcal{M}^{2(1)} T_{c}^{b},{ }^{(1)} f_{b c}\right),
$$

where $\mathrm{L}_{a}$ is a linear operator. The replacement principle for the divergence of the stress-energy tensor states that the gauge invariants associated with ${ }^{(1)} I_{a},{ }^{(1)} T_{b}^{a}$ and (1) $f_{a b}$ by $X$-compensation are related by the same linear operator:

$$
\mathbf{I}_{a}[X]=\mathrm{L}_{a}\left(\mathbf{T}_{c}^{b}[X], \mathbf{f}_{b c}[X]\right)
$$

for any gauge field $X$. If the stress-energy tensor is conserved at zero order (i.e. $I_{a}(0)=0$ then ${ }^{(1)} I_{a}$ is a gauge invariant, and the left sides of (100) and (101) are equal.

This result is adapted from Nakamura (2005) (see equations (3.90), (3.91) and (3.20)). Use of the Replacement Principle in Appendix B makes the transition from gauge-variant to gauge-invariant equations particularly easy and transparent.

\section{B Derivation of the conservation equations}

In this Appendix we give the derivation of the linearized conservation equations in the form (43), using the methods developed in UW (see in particular Section 2 and

\footnotetext{
${ }^{38}$ In the usual derivation one first obtains a system of partial differential equations for the spatial gradients of the energy density and the Hubble scalar, and then one takes the spatial divergence to obtain partial differential equations for scalars, a more lengthy process. See, for example, BDE.
} 
Appendix B). We express the covariant derivative ${ }^{\epsilon} \boldsymbol{\nabla}_{a}$ of the metric $g_{a b}(\epsilon)$ in terms of the covariant derivative ${ }^{0} \bar{\nabla}_{a}$ of the conformal background metric $\gamma_{a b}$ as follows:

$$
{ }^{\epsilon} \nabla_{a} A_{c}^{b}(\epsilon)={ }^{0} \bar{\nabla}_{a} A_{c}^{b}(\epsilon)+Q_{a d}^{b}(\epsilon) A_{c}^{d}(\epsilon)-Q_{a c}^{d}(\epsilon) A_{d}^{b}(\epsilon) .
$$

The object $Q_{b c}^{a}(\epsilon)$ is written as the sum of two parts:

$$
Q_{b c}^{a}(\epsilon)=\bar{Q}_{b c}^{a}(\epsilon)+\tilde{Q}_{b c}^{a}(\epsilon)
$$

where

$$
\begin{aligned}
& \bar{Q}_{b c}^{a}(\epsilon):=2 \delta^{a}{ }_{(b} r_{c)}-\bar{g}^{a d}(\epsilon) \bar{g}_{b c}(\epsilon) r_{d}, \quad \text { with } \quad r_{a}:={ }^{0} \bar{\nabla}_{a}(\ln a), \\
& \tilde{Q}_{b c}^{a}(\epsilon):=\frac{1}{2} \bar{g}^{a d}(\epsilon)\left({ }^{0} \bar{\nabla}_{c} \bar{g}_{d b}(\epsilon)-{ }^{0} \bar{\nabla}_{d} \bar{g}_{b c}(\epsilon)+{ }^{0} \bar{\nabla}_{b} \bar{g}_{c d}(\epsilon)\right) .
\end{aligned}
$$

It follows from (104a) and (104b), in conjunction with ${ }^{0} \bar{\nabla}_{a} \gamma_{b c}=0$, that at zeroth and first order we obtain

$$
\begin{array}{ll}
{ }^{(0)} \bar{Q}_{b c}^{a}=2 \delta^{a}{ }_{(b} r_{c)}-\gamma^{a d} \gamma_{b c} r_{d}, & { }^{(0)} \tilde{Q}_{b c}^{a}=0 \\
{ }^{(1)} \bar{Q}_{b c}^{a}=\left(f^{a d} \gamma_{b c}-\gamma^{a d} f_{b c}\right) r_{d}, & { }^{(1)} \tilde{Q}_{b c}^{a}=\frac{1}{2} \gamma^{a d}\left({ }^{0} \bar{\nabla}_{c} f_{d b}-{ }^{0} \bar{\nabla}_{d} f_{b c}+{ }^{0} \bar{\nabla}_{b} f_{c d}\right) .
\end{array}
$$

Consider tensors $A_{b}^{a}(\epsilon)$ such that $\lambda^{2} A_{b}^{a}(\epsilon)$ is dimensionless, where $\lambda>0$ is a background quantity with dimension length. As follows from (102), the equation $0=\lambda^{2} \epsilon \nabla_{b} A_{a}^{b}(\epsilon)$ can be written as

$$
0=\left({ }^{0} \bar{\nabla}_{b}-2 s_{b}\right) \lambda^{2} A_{a}^{b}(\epsilon)+2 Q_{b[c}^{c}(\epsilon) \lambda^{2} A_{a]}^{b}(\epsilon),
$$

where

$$
s_{a}:={ }^{0} \bar{\nabla}_{a}(\ln \lambda),
$$

which yields the following zeroth and first order expressions

$$
\begin{aligned}
& 0=\left({ }^{0} \bar{\nabla}_{b}-2 s_{b}\right) \lambda^{2(0)} A_{a}^{b}+2^{(0)} \bar{Q}_{b[c}^{c} \lambda^{2(0)} A_{a]}^{b}, \\
& 0=\left({ }^{0} \bar{\nabla}_{b}-2 s_{b}\right) \lambda^{2(1)} A_{a}^{b}+2^{(0)} \bar{Q}_{b[c}^{c} \lambda^{2(1)} A_{a]}^{b}+2^{(1)} Q_{b[c}^{c} \lambda^{2(0)} A_{a]}^{b} .
\end{aligned}
$$

We now specialize $A_{b}^{a}(\epsilon)$ to a stress-energy tensor $T_{b}^{a}(\epsilon)$ that obeys the background symmetries, i.e. that satisfies (13), and choose the normalizing factor $\lambda$ as in equation (15), i.e. $\lambda=\mathcal{M}$. We also assume that $T_{b}^{a}(\epsilon)$ satisfies a conservation law of the form ${ }^{\epsilon} \nabla_{b} T_{a}^{b}(\epsilon)=0$. Relative to local coordinates we obtain

$$
r_{\alpha}=\mathcal{H} \delta^{0}{ }_{\alpha}, \quad s_{\alpha}=\frac{3}{2} \mathcal{H}\left(1+\mathcal{C}_{T}^{2}\right) \delta_{\alpha}^{0} .
$$

On substituting from (105) the zeroth order expression (108a) yields equations (14), and the temporal and spatial components of the first order expression (108b) assume the following form:

$$
\begin{aligned}
& 0=\partial_{\eta}\left(\mathcal{M}^{2(1)} T_{0}^{0}-\frac{1}{2} f_{i}^{i}\right)+\mathbf{D}_{i}\left(\mathcal{M}^{2(1)} T_{0}^{i}\right)-\mathcal{H} \mathcal{M}^{2}\left({ }^{(1)} T_{i}^{i}+3 \mathcal{C}_{T}^{2(1)} T_{0}^{0}\right) \\
& 0=\left(\partial_{\eta}-3 \mathcal{H} \mathcal{C}_{T}^{2}\right)\left(\mathcal{M}^{2(1)} T_{i}^{0}\right)+\mathbf{D}_{j}\left(\mathcal{M}^{2(1)} T_{i}^{j}\right)-\mathcal{H} \gamma_{i j} \mathcal{M}^{2(1)} T_{0}^{j}-\frac{1}{2} \mathbf{D}_{i} f_{00}+\mathcal{H} f_{0 i}
\end{aligned}
$$


We simplify these equations by first expressing ${ }^{(1)} T_{0}^{i}$ in terms of ${ }^{(1)} T_{i}^{0}$ :

$$
\mathcal{M}^{2(1)} T_{0}^{i}=-\gamma^{i j}\left(\mathcal{M}^{2(1)} T_{j}^{0}-f_{0 j}\right),
$$

and decomposing ${ }^{(1)} T_{j}^{i}$ into its tracefree part and its trace using (18). We then introduce the intrinsic gauge invariants $\hat{\mathbb{T}}_{i}^{j}, \mathbb{T}_{i}$ and $\mathbb{T}$ as defined by (17), expressing the trace ${ }^{(1)} T_{i}^{i}$ in terms of $\mathbb{T}$. As a result of these changes equations (110) yield:

$$
\begin{aligned}
& 0=\partial_{\eta}\left(\mathcal{M}^{2(1)} T_{0}^{0}-\frac{1}{2} f_{i}^{i}\right)-\mathbf{D}^{i}\left(\mathcal{M}^{2(1)} T_{i}^{0}-f_{0 i}\right)-3 \mathcal{H} \mathbb{T}, \\
& 0=\left(\partial_{\eta}+\mathcal{H}\right)\left(\mathcal{M}^{2}{ }^{(1)} T_{i}^{0}\right)-\frac{1}{2} \mathbf{D}_{i} f_{00}+\mathcal{C}_{T}^{2} \mathbb{T}_{i}+\mathbf{D}_{j} \hat{\mathbb{T}}^{j}{ }_{i}+\mathbf{D}_{i} \mathbb{T} .
\end{aligned}
$$

We now apply the Replacement Principle to these equations, which entails performing the following replacements:

$$
\begin{aligned}
f_{00} & \rightarrow \mathbf{f}_{00}[X], \quad f_{0 i} \rightarrow \mathbf{f}_{0 i}[X], \quad f_{i j} \rightarrow \mathbf{f}_{i j}[X], \\
\mathcal{M}^{2}{ }^{(1)} T_{0}^{0} & \rightarrow \mathbb{T}_{0}^{0}[X], \quad \mathcal{M}^{2(1)} T_{i}^{0} \rightarrow \mathbb{T}_{i}^{0}[X] .
\end{aligned}
$$

On substituting from (8) and (21) and noting that

$$
\mathbf{D}^{i} \mathbf{f}_{0 i}[X]=\mathbf{D}^{2} \mathbf{B}[X], \quad \mathbf{D}^{i} \mathbb{T}_{i}^{0}[X]=\mathbf{D}^{2} \mathbb{V}[X]
$$

equation (112a) assumes the form (43a). After performing the replacements (113) in (112b) we apply the operator $\mathbf{D}^{i}$ in order to extract the scalar mode. We then substitute from (8) and (21), noting (114) and the fact that 39

$$
\mathbf{D}^{i} \mathbb{T}_{i}=\mathbf{D}^{2} \mathbb{D}, \quad \mathbf{D}^{i} \mathbf{D}_{j} \hat{\mathbb{T}}_{i}^{j}=\mathbf{D}^{i} \mathbf{D}_{j} \mathbf{D}_{i}^{j} \bar{\Pi}=\mathbf{D}^{2} \bar{\Xi},
$$

where $\bar{\Xi}$ is defined by (43c). The result is that (112b) assumes the form $\mathbf{D}^{2} \mathbb{C}=0$. Since we are assuming, as in UW, that the inverse operator of $\mathbf{D}^{2}$ exists, we obtain $\mathbb{C}=0$, which is precisely the desired equation (43b).

\section{Derivation of the $1+3$ perturbation equations}

\section{C.1 Derivation of the evolution equations}

\section{The evolution equation for $D$}

We begin with the conservation equations for the stress-energy tensor (82), linearized by dropping products of first order quantities40:

$$
\begin{aligned}
\rho^{\prime} & =-3 a H(\rho+p)-\tilde{\mathbf{D}}^{a} q_{a}, \\
h_{a}^{b} q_{b}^{\prime} & =-4 a H q_{a}-\tilde{\mathbf{D}}_{a} p-(\rho+p) a \dot{u}_{a}-\tilde{\mathbf{D}}_{b} \pi_{a}^{b} .
\end{aligned}
$$

\footnotetext{
${ }^{39}$ The third equality follows from the identity (B.39e) in UW.

${ }^{40}$ See, for example, Wainwright and Ellis (1997), equations (1.48) and (1.49), after multiplying by $a$ to change the dot derivative to prime.
} 
In these equations the differential operators ' and $\tilde{\mathbf{D}}_{a}$ are defined by (87) and (89). We require the zero order version of (116a) which we write in the form 41

$$
\mathcal{M}^{2(0)} \rho^{\prime}=-3 \mathcal{H}
$$

which leads to the evolution equation for $\mathcal{M}^{2}$ :

$$
\left(\mathcal{M}^{2}\right)^{\prime}=3\left(1+\mathcal{C}_{T}^{2}\right) \mathcal{H} \mathcal{M}^{2}
$$

We apply the operator $\mathcal{M}^{2} \tilde{\mathbf{D}}^{2}$ to (116a) and the operator $\mathcal{M}^{2} \tilde{\mathbf{D}}^{a}$ to (116b) and then linearize, obtaining

$$
\begin{aligned}
D^{\prime}-3 \mathcal{H} \mathcal{C}_{T}^{2} D+Z & =-3 \mathcal{H}\left(\tilde{\mathbf{D}}^{a}\left(a \dot{u}_{a}\right)+P\right)-\tilde{\mathbf{D}}^{2} \tilde{Q}, \\
\tilde{\mathbf{D}}^{a}\left(a \dot{u}_{a}\right)+P & =-(\tilde{\Upsilon}+\tilde{\Pi})
\end{aligned}
$$

On substituting (119b) in (119a) we obtain the evolution equation (86a) for $D$.

In deriving (119a) and (119b) we use the following linearized commutativity properties:

$$
\tilde{\mathbf{D}}^{2}\left(A^{\prime}\right)=\left(\tilde{\mathbf{D}}^{2} A\right)^{\prime}-{ }^{(0)} A^{\prime}\left(\tilde{\mathbf{D}}^{a}\left(a \dot{u}_{a}\right)\right),
$$

where $A$ is any scalar field, and

$$
\tilde{\mathbf{D}}_{a} A_{b}^{\prime}=\left(\tilde{\mathbf{D}}_{a} A_{b}\right)^{\prime}
$$

where $A_{a}$ is any covariant vector field. In differentiating products of perturbed quantities such as $\rho H, H q_{a}$ and $(\rho+p) \dot{u}_{a}$ we use the following expansion to linear order:

$$
A B={ }^{(0)} A B+{ }^{(0)} B A-{ }^{(0)} A^{(0)} B,
$$

where $A$ and $B$ are geometric quantities with background values ${ }^{(0)} A$ and ${ }^{(0)} B$, one of which may be zero.

\section{The evolution equation for $Z$}

We begin with the linearized Raychaudhuri equation written in the form

$$
3\left(a H^{\prime}+a^{2} H^{2}\right)-\tilde{\mathbf{D}}^{a}\left(a \dot{u}_{a}\right)+\frac{1}{2} a^{2}(\rho+3 p)=0 .
$$

We use (121) with $A=B=H$ to write $a^{2} H^{2}=2 \mathcal{H}(a H)-\mathcal{H}^{2}$, where $\mathcal{H}:=a^{(0)} H$, and use (119b) to eliminate $\dot{u}_{a}$. We then apply the operator $\tilde{\mathbf{D}}^{2}$ to (122). After using (120a) and the definitions of $D, Z$ and $P$ we obtain 42

$$
Z^{\prime}+\mathcal{H} Z+\frac{1}{2} \mathcal{A} D=-\left(\tilde{\mathbf{D}}^{2}+3 K\right) P-\left(\tilde{\mathbf{D}}^{2}+3 K-\frac{3}{2} \mathcal{A}\right)(\tilde{\Upsilon}+\tilde{\Pi})
$$

In deriving this equation we have also used (37) and (137). We finally use (90) to express $P$ in (123) in terms of $D$ and $\tilde{\Gamma}$, which gives the evolution equation (86b) for $Z$.

\footnotetext{
${ }^{41}$ For a background scalar ${ }^{(0)} A^{\prime}$ is the ordinary derivative with respect to conformal time $\eta$.

${ }^{42}$ In doing calculations such as these one should keep in mind that ${ }^{(3)} \nabla_{a}\left(a^{\prime}\right) \neq 0$, where ' is defined by (87), even though we have chosen $a$ to be the background scale factor $\left(\right.$ i.e. ${ }^{(3)} \nabla_{a}(a)=0$.
} 


\section{C.2 Relation between the $1+3$ and the metric-based ap- proaches}

\section{Fundamental 4-velocity and energy flow vector}

We begin with the decomposition of the stress-energy tensor with respect to a unit timelike vector field $u^{a}$, which is given by (82). The Stewart-Walker lemma implies that the linear perturbation ${ }^{(1)} q_{a}$ is a gauge invariant. Since ${ }^{(0)} u^{a}=a^{-1} \delta_{0}^{a}$ and ${ }^{(0)} u_{a}=-a \delta_{a}^{0}$, it follows that ${ }^{(1)} q_{0}=0$, and hence that

$$
{ }^{(1)} T_{0}^{0}=-{ }^{(1)} \rho, \quad \mathcal{M}^{2}{ }^{(1)} T_{i}^{0}=v_{i}+\overline{\mathbb{Q}}_{i},
$$

where

$$
a v_{i}:={ }^{(1)} u_{i}, \quad a \overline{\mathbb{Q}}_{i}:=\mathcal{M}^{2}{ }^{(1)} q_{i} .
$$

It follows from (124) and (19b) that

$$
\mathbb{T}_{i}^{0}[X]=\mathbf{v}_{i}[X]+\overline{\mathbb{Q}}_{i}
$$

where

$$
\mathbf{v}_{i}[X]=v_{i}+\mathbf{D}_{i} X^{0}
$$

We decompose $\overline{\mathbb{Q}}_{i}, \mathbf{v}_{i}$ and $v_{i}$ according to

$$
\overline{\mathbb{Q}}_{i}=\mathbf{D}_{i} \overline{\mathbb{Q}}+\tilde{\mathbb{Q}}_{i}, \quad \mathbf{v}_{i}[X]=\mathbf{D}_{i} \mathbf{v}[X]+\tilde{\mathbf{v}}_{i}, \quad v_{i}=D_{i} v+\tilde{v}_{i},
$$

with

$$
\mathbf{D}^{i} \tilde{\mathbb{Q}}_{i}=0, \quad \mathbf{D}^{i} \tilde{\mathbf{v}}_{i}=0, \quad \mathbf{D}^{i} \tilde{v}_{i}=0
$$

It now follows from (21d $)$, (125a ) and (126) that

$$
\mathbb{V}[X]=\mathbf{v}[X]+\overline{\mathbb{Q}}, \quad \mathbb{V}_{i}=\tilde{\mathbf{v}}_{i}+\tilde{\mathbb{Q}}_{i}
$$

Thus if the preferred timelike vector field $u^{a}$ is an eigenvector of the stress-energy tensor, i.e. if the energy transfer vector $q^{a}$ is zero, then the stress-energy gauge invariants $\mathbb{V}[X]$ and $\mathbb{V}_{i}$ equal the gauge invariants $\mathbf{v}[X]$ and $\tilde{\mathbf{v}}_{i}$ associated with $u^{a}$. In addition it follows from (125b) and (126) that

$$
\mathbf{v}[X]=v+X^{0}, \quad \tilde{\mathbf{v}}_{i}=\tilde{v}_{i} .
$$

\section{Spatial gradient and Laplacian of a scalar}

We have seen that the $1+3$ approach to cosmological perturbations is based on the spatial gradient and Laplacian of the density $\rho$ and the Hubble scalar $H$. We now define these quantities for a scalar field of given dimension, using a background normalization factor $\lambda$ of dimension length. Let $f$ be a scalar such that $\lambda^{n} f$ is dimensionless, and whose unperturbed value is a function only of $\eta$. We define the dimensionless spatial gradient and spatial Laplacian of $f$ according to

$$
F_{a}:=\frac{1}{a} \tilde{\mathbf{D}}_{a}\left(\lambda^{n} f\right), \quad F:=\tilde{\mathbf{D}}^{2}\left(\lambda^{n} f\right)
$$


using the notation (85)).

Our goal is to relate the linear perturbation of $F_{a}$ and $F$ to the linear perturbation of $f$. Regarding all perturbed quantities as functions of the perturbation parameter $\epsilon$, we write

$$
F_{a}(\epsilon):=h_{a}^{b}(\epsilon)^{\epsilon} \nabla_{a}\left(\lambda^{n} f(\epsilon)\right), \quad F(\epsilon):=a^{2} g^{a b}(\epsilon) h_{a}^{c}(\epsilon)^{\epsilon} \nabla_{c} F_{b}(\epsilon),
$$

with $f(\epsilon)={ }^{(0)} f+\epsilon^{(1)} f+\ldots$, etc. A straightforward calculation yield\& 43

$$
\begin{aligned}
& { }^{(0)} F_{a}=0, \quad{ }^{(1)} F_{0}=0, \quad{ }^{(1)} F_{i}=\lambda^{n}\left(\mathbf{D}_{i}{ }^{(1)} f+{ }^{(0)} f^{\prime} v_{i}\right), \\
& { }^{(0)} F=0, \quad{ }^{(1)} F=\mathbf{D}^{i(1)} F_{i} .
\end{aligned}
$$

We note that the background values ${ }^{(0)} F_{a}$ and ${ }^{(0)} F$ are zero due to our assumption that ${ }^{(0)} f={ }^{(0)} f(\eta)$. On account of the Stewart-Walker lemma the linear perturbations ${ }^{(1)} F_{i}$ and ${ }^{(1)} F$ are gauge-invariant. We can write them in a manifestly gauge-invariant form by noting that

$$
\lambda^{n}\left(\mathbf{D}_{i}^{(1)} f+{ }^{(0)} f^{\prime} v_{i}\right)=\mathbf{D}_{i} \mathbf{f}[X]+\lambda^{n(0)} f^{\prime} \mathbf{v}_{i}[X],
$$

where $\mathbf{f}[X]$ is the gauge invariant associated with $f$ by $X$-compensation and $\mathbf{v}_{i}[X]$ is given by (125b). It follows from (131), (132) and (126) that

$$
{ }^{(1)} F=\mathbf{D}^{2}\left(\mathbf{f}[X]+\lambda^{n(0)} f^{\prime} \mathbf{v}[X]\right) .
$$

For future use we choose $X=X_{\mathrm{v}}$ in (133) and use the fact that $\mathbf{v}\left[X_{\mathrm{v}}\right]=-\overline{\mathbb{Q}}$, as follows from (127). Equation (133) assumes the form

$$
{ }^{(1)} F=\mathbf{D}^{2}\left(\mathbf{f}\left[X_{\mathrm{v}}\right]-\lambda^{n(0)} f^{\prime} \overline{\mathbb{Q}}\right) .
$$

\section{Relation between the variables}

We need an expression for the gauge-invariant linear perturbation $\mathbf{H}[X]$ of the Hubble scalar $H$ of the preferred congruence, which is defined by

$$
\mathbf{H}[X]=a\left({ }^{(1)} H-{ }^{(0)} H^{\prime} X^{0}\right),
$$

in accordance with the general definition (3). It follows from the expression (B.41a) for $a^{(1)} H$ in UW, in conjunction with (9a) and (128), that 44

$$
\mathbf{H}[X]=\frac{1}{3} \mathbf{D}^{2}(\mathbf{v}[X]-\mathbf{B}[X])-\left(\partial_{\eta} \Psi[X]+\mathcal{H} \Phi[X]\right) .
$$

We have also used the fact that

$$
a^{(0)} H^{\prime}=\mathcal{H}^{\prime}-\mathcal{H}^{2}=-\left(\frac{1}{2} \mathcal{A}_{G}-K\right),
$$

the second equality following from (36) $)$.

\footnotetext{
${ }^{43}$ We note that ${ }^{\epsilon} \nabla_{a} A(\epsilon)={ }^{0} \bar{\nabla}_{a} A(\epsilon)$ for a scalar A, and that ${ }^{0} \bar{\nabla}_{i} A=\mathbf{D}_{i} A$ and ${ }^{0} \bar{\nabla}_{0} A=\partial_{\eta} A$.

${ }^{44}$ This is another example of a Replacement Principle, where an equation remains valid when each gauge-variant quantity is replaced by the associated gauge invariant defined using $X$-compensation.
} 
First we note that

$$
\mathbf{v}[X]-\mathbf{B}[X]=\mathbb{V}[X]-\mathbf{B}[X]-\overline{\mathbb{Q}}=\mathbb{V}-\overline{\mathbb{Q}},
$$

as follows from (127) and the $X$-independent gauge invariant $[\mathbb{V}, \mathbf{B}]$ in (29). Second we can use the transition rules (28) and (32) to show that the gauge invariant

$$
\partial_{\eta} \Psi[X]+\mathcal{H} \Phi[X]+\left(-\mathcal{H}^{\prime}+\mathcal{H}^{2}\right) \mathbb{V}[X],
$$

is $X$-independent. Evaluating this expression for $X^{0}=X_{\mathrm{v}}^{0}$ and $X^{0}=X_{\mathrm{p}}^{0}$ yields

$$
\partial_{\eta} \Psi_{\mathrm{v}}+\mathcal{H} \Phi_{\mathrm{v}}=-K \mathbb{V}
$$

where we have used the linearized Einstein equation (40d), the background Einstein equation $\mathcal{A}_{T}=\mathcal{A}_{G}$ and the definition (36) of $\mathcal{A}_{G}$. Finally we choose $X^{0}=X_{\mathrm{v}}^{0}$ in (136) and substitute from (138) and (140) to obtain the desired result that

$$
3 \mathbf{H}_{\mathrm{v}}=\left(\mathbf{D}^{2}+3 K\right) \mathbb{V}-\mathbf{D}^{2} \overline{\mathbb{Q}}=\mathbb{Z}-\mathbf{D}^{2} \overline{\mathbb{Q}},
$$

the second equality following from (48).

We can now use (134) to relate the perturbations of the variables $D$ and $Z$ in the $1+3$ approach to the corresponding variables $\mathbb{D}$ and $\mathbb{Z}$ in the metric-based approach. First choose $f=\rho, \lambda=\mathcal{M}$ and $n=2$ in (134), and use (124), (117), (19a) and (35b) to obtain

$$
{ }^{(1)} D=\mathbf{D}^{2}(\mathbb{D}+3 \mathcal{H} \overline{\mathbb{Q}}) .
$$

Second, choose $f=H, \lambda=a$ and $n=1$ in (134). Equations (141) and (137) then lead te 45

$$
{ }^{(1)} Z=\mathbf{D}^{2}\left(\mathbb{Z}-\left(\mathbf{D}^{2}+3 K-\frac{3}{2} \mathcal{A}_{G}\right) \overline{\mathbb{Q}}\right) .
$$

We also need the relation

$$
{ }^{(1)} \tilde{Q}=\mathbf{D}^{2} \overline{\mathbb{Q}},
$$

which can be derived from the definition (88) of $\tilde{Q}$ and the definition (126) of $\overline{\mathbb{Q}}$. The desired equations (98a) and (98b) now follow immediately from (142) and (143) in conjunction with (144). Further, equations (98c) can be derived from the definitions (90) and (88) of $\tilde{\Gamma}$ and $\tilde{\Pi}$. Finally, equation (97) can be derived in a similar manner using (96) and the footnote following (130).

\section{References}

Bardeen, J. M. (1980) Gauge-invariant cosmological perturbations, Phys. Rev. D 22, 1882-1905.

Bardeen, J. M. (1988) Cosmological perturbations, from quantum fluctuations to large scale structure, in Cosmology and Particle Physics, edited by Li-Zhi Fang and

\footnotetext{
${ }^{45}$ Note the factor 3 in the definition of $Z$ in (84) compared to (129), so that $Z=3 F$ and ${ }^{(1)} Z=3^{(1)} F$.
} 
A. Zee, pages 1-64 (Gordon and Breach, New York).

Bardeen, J.M., Steinhardt, P.J. and Turner, M.S. (1983) Spontaneous creation of almost scale-free density perturbations in an inflationary universe, Phys. Rev. D 28, 679-693.

Brandenberger, R. and Khan, R. (1984) Cosmological perturbations in inflationaryuniverse models, Phys. Rev. D 29, 2172-2190.

Bruni, M., Dunsby, P.K.S. and Ellis, G.F.R. (1992a) Cosmological perturbations and the meaning of gauge-invariant variables, Astrophysical J. 395, 34-53.

Bruni, M., Dunsby, P.K.S. and Ellis, G.F.R. (1992b) Gauge-invariant perturbations in a scalar field dominated universe, Class. Quantum Grav. 9, 921-945.

Bruni, M., Matarrese, S., Mollerach, S., and Sonego, S. (1997) Perturbations of spacetime: gauge transformations and gauge-invariance at second order and beyond, Class. Quantum Grav. 14, 2585-2606.

Dunsby, P.K.S., Bruni, M., and Ellis, G.F.R. (1992) Covariant perturbations in a multifluid cosmological medium, Astrophysical J. 395, 54-74.

Durrer, R. (2008) The Cosmic Microwave Background, Cambridge University Press.

Ellis, G.F.R. and Bruni, M. (1989), Covariant and gauge-invariant approach to cosmological density fluctuations, Phys. Rev. D 40, 1804-1818.

Ellis, G.F.R., Bruni, M. and Hwang, J (1990) Density-Gradient-vorticity relation in perfect-fluid Robertson-Walker perturbations, Phys. Rev. D 42, 1035-1046.

Ellis, G.F.R., Hwang, J. and Bruni, M. (1989) Covariant and gauge-independent perfect fluid Robertson-Walker perturbations, Phys. Rev. D 40, 1819-1826.

Hawking, S.W. (1966) Perturbations of an expanding universe, Astrophysical J. 145, 544-54.

Hwang, J. (1991) Perturbations of the Robertson-Walker space: multicomponent sources and generalized gravity, Astrophysical J. 375, 443-462.

Hwang, J. and Noh, H. (1999) Relativistic Hydrodynamic Cosmological Perturbations, General Relativity and Gravitation 31,1131-1146.

Kodama, H. and Sasaki, M. (1984) Cosmological Perturbation Theory, Prog. Theoret. Phys. Suppl. 78,1-166.

Langlois, D. and Vernizzi, F. (2005) Conserved nonlinear quantities in cosmology, 
Phys. Rev. D 72, 103501 (1-9).

Malik, K. A. and Wands, D. (2009) Cosmological perturbations, Physics Reports 475, 1-51.

Mukhanov, V. (2005) Physical Foundations of Cosmology, Cambridge University Press.

Mukhanov, V. F., Feldman, H. A. and Brandenberger, R. H. (1992) Theory of cosmological perturbations, Physics Reports 215, 203-333.

Nakamura, K. (2003) Gauge Invariant Variables in Two-Parameter Nonlinear Perturbations, Prog. Theor. Phys. 110, 723-755.

Nakamura, K. (2005) Second Order Gauge Invariant Perturbation Theory, Prog. Theor. Phys. 113, 481-511.

Nakamura, K. (2007) Second Order Gauge Invariant Cosmological Perturbation Theory, Prog. Theor. Phys. 117, 17-74.

Tsagas C. G., Challinor A. and Maartens R. (2008) Relativistic cosmology and largescale structure, Physics Reports 465, 61-147.

Uggla, C. and Wainwright, J. (2011) Cosmological Perturbation Theory Revisited, Class. Quantum Grav. 28, 175017(26pp).

Wainwright, J. and Ellis, G.F.R. (1997) Dynamical Systems in Cosmology, Cambridge University Press.

Wands, D. Malik, K. A. Lyth, D. H. and Liddle, A. R. (2000), New approach to the evolution of cosmological perturbations on large scales, Phys. Rev. D 62, 043527 (1-8).

Weinberg, S. (2008) Cosmology, Oxford University Press.

Woszczyna, A. and Kulak, A. (1989) Cosmological perturbations - extension of Olson's gauge-invariant method, Class. Quantum Grav. 6, 1665-1671. 